\title{
A BEGINNER'S GUIDE TO EDGE AND COVER IDEALS
}

\author{
ADAM VAN TUYL
}

Abstract. Our goal is to introduce the basics properties of edge and cover ideals, and to introduce some current research themes. We also include an introduction to the Macaulay 2 computer package EdgeIdeals. These notes are an expanded version of my lectures given at MONICA: MONomial Ideals, Computations and Applications, at the CIEM, Castro Urdiales (Cantabria, Spain) in July 2011.

\section{Contents}

Introduction 2

1. The basics of edge and cover ideals 3

1.1. Definitions 3

1.2. Colouring Graphs: an illustration of the dictionary 5

\begin{tabular}{lll}
\hline 1.3. & Associated Simplicial Complexes & 10
\end{tabular}

$\begin{array}{ll}\text { 1.4. Additional constructions } & 13\end{array}$

2. $\quad$ Splitting monomial ideals and Fröberg's Theorem 14

\begin{tabular}{lll}
\hline 2.1. & Splitting Monomial Ideals & 16
\end{tabular}

2.2. Proof of Fröberg's Theorem 19

2.3. Additional comments and open questions 21

3. Colouring graphs and decomposing cover ideals 23

3.1. Powers of cover ideals: associated primes 24

$\begin{array}{lll}3.2 . & \text { Powers of cover ideals: irreducible decomposition } & 27\end{array}$

3.3. Persistence of primes and a conjecture 29

4. The Macaulay 2 package EdgeIdeals 30

4.1. Getting Started 31

4.2. The EdgeIdeals Package 31

5. Tutorials 36

5.1. Tutorial 1: Splitting Monomial Ideals 36

5.2. Tutorial 2: Regularity 38

\begin{tabular}{ll}
\hline References & 39
\end{tabular}

(Submitted Version; February 13, 2012). 


\section{INTRODUCTION}

Monomial ideals, although intrinsically interesting, play an important role in studying the connections between commutative algebra and combinatorics. Broadly speaking, problems in combinatorics are encoded into monomial ideals, which then allow us to use techniques and methods in commutative algebra to solve the original question. Stanley's proof of the Upper Bound Conjecture [59] for simplicial spheres is seen as one of the early highlights of exploiting this connection between two fields. To bridge these two areas of mathematics, Stanley used square-free monomial ideals.

Over the last decade or so, commutative algebraists have become interested in studying the properties of finite simple graphs through monomial ideals. Fröberg [30], Villarreal [63], and Simis, Vasconcelos, and Villarreal [57] were among the early pioneers in this field. The starting point of these projects is to use the edges of a finite simple graph to construct a monomial ideal, usually called the edge ideal, and to study the properties of this monomial ideal using the properties of the graph, and vice versa. In these notes, we provide an introduction to the edge ideal and the cover ideal, two monomial ideals that can be constructed from a finite simple graph $G$ using its edges, and discuss some current research themes related to these ideals.

Section 1 is devoted to the basics of edge and cover ideals. We start with the definition of these ideals, and work out some of their basic properties. One of the themes in the study of edge and cover ideals is to build a dictionary between graph theory and commutative algebra. To illustrate this dictionary, we explain how the chromatic number of a graph is encoded algebraically. We also describe how Stanley-Reisner theory can give us information about these ideals.

The goal of Section 2 is to introduce the technique of splitting monomial ideals. Splitting a monomial ideal is a technique that originates in a paper of Eliahou and Kervaire [18]. Roughly speaking, a splitting of a monomial ideal allows us to describe its graded Betti numbers in terms of the graded Betti numbers of smaller ideals. Over the last couple of years, this method has proved useful in a number of contexts. We introduce this machinery, and as an application, we prove Fröberg's [30] characterization of edge ideals with a linear resolution.

In Section 3, we look at decompositions of powers of the cover ideal. In the first part, we show that some of the associated primes of the powers of the cover ideal correspond to colouring information about the associated graph. In the second part, we turn our attention to the irreducible ideals in the irreducible decomposition of the powers of cover ideals and relate their information to colouring information about the graph. We end with a conjecture about the persistence of associated primes for cover ideals.

At the end of these notes, we provide a brief introduction to the Macaulay 2 package EdgeIdeals [29], written by C. Francisco, A. Hoefel, and the author. It is hoped that this package will facilitate your own research. We have also include two tutorials that 
will allow you to start exploring edge and cover ideals using Macaulay 2. The tutorials include a number of open problems.

When preparing these notes, I have assumed that the reader is familiar with the basics of Stanley-Reisner ideals, minimal free graded resolutions, and associated primes of ideals. If you need brushing up on some of these topics, I would like to point the reader to [4, 36, 49, 54, 56, 64. I have not assumed any previous knowledge about graph theory. Instead, I will introduce any terms as needed. However, you may want to have a graph theory textbook handy as you read through these notes.

Given the wealth of research on edge and cover ideals, these notes cannot do adequate justice to this topic. For example, I had no time to look at the interesting problem of determining when a graph is Cohen-Macaulay or sequentially Cohen-Macaulay. My hope is to whet your appetite, and let you explore the field.

If you are interested in learning more, I would recommend that your reading list include Villarreal's textbook [64] (especially Chapter 6), Herzog and Hibi's text [36] (in particular, Chapter 9), the recent survey of Morey and Villarreal [50], and an older survey of Hà and the author [33]. As an aide to help you develop your own projects, scattered throughout the notes are a number of open questions. I would also encourage you to come with your own questions; one approach is to simply browse a book on graph theory or the latest issue of a graph theory journal and ask yourself if a particular problem or result can be rephrased as an algebraic result. Have fun!

Acknowledgements. I would like to thank the organizers of MONICA, Anna M. Bigatti, Philippe Gimenez, and Eduardo Sáenz-de-Cabezón, for the invitation to participate in this conference. As well, I would like to thank all the participants for stimulating discussions and their feedback. I would also like to thank Ben Babcock, Ashwini Bhat, Jen Biermann, Chris Francisco, Tai Hà, Andrew Hoefel, and Ştefan Tohăneanu for their feedback on preliminary drafts. The author was supported in part by an NSERC Discovery Grant.

\section{The BASICS OF EDGE AND COVER IDEALS}

In this section, we introduce edge and cover ideals. One theme in the study of these ideals is to understand how graph theoretic invariants are encoded algebraically in these ideals. We illustrate this theme by using the chromatic number of a graph as a case study. Because edge and cover ideals are square-free monomial ideals, one can also apply the theory of Stanley-Reisner ideals and simplicial complexes to study these ideals. Using results from Stanley-Reisner theory, we identify other graph theoretic invariants encoded in these ideals. Furthermore, we prove that the edge and cover ideals are dual to each other with respect to Alexander duality.

1.1. Definitions. Our basic combinatorial object will be a finite simple graph. A finite graph $G$ is a pair $G=(V(G), E(G))$ where $V(G)=\left\{x_{1}, \ldots, x_{n}\right\}$ is the set of vertices of $G$, and $E(G)$ is a collection of two element subsets of $V(G)$, usually called the edges of 
$G$. A finite graph is simple if we do not allow multiple edges between vertices and we do not allow loops at vertices, i.e., an edge from a vertex $x_{i}$ to itself.

Example 1.1. The following pair is a finite simple graph:

$$
G=\left(\left\{x_{1}, \ldots, x_{5}\right\},\left\{\left\{x_{1}, x_{2}\right\},\left\{x_{2}, x_{3}\right\},\left\{x_{3}, x_{4}\right\},\left\{x_{4}, x_{5}\right\},\left\{x_{5}, x_{1}\right\}\right\}\right) .
$$

It is standard practise to represent a graph as a figure. More precisely, we introduce a node for each vertex $x \in V(G)$. We then join two vertices $x_{i}$ and $x_{j}$ by a line segment if and only if $\left\{x_{i}, x_{j}\right\} \in E(G)$. Thus, the above graph $G$ can be represented as the following figure:

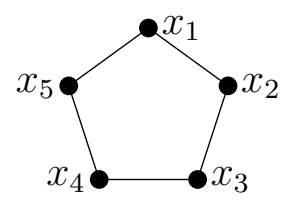

The figure below is not a finite simple graph since it has a loop at the vertex $x_{1}$, and three edges from $x_{4}$ to $x_{5}$ :

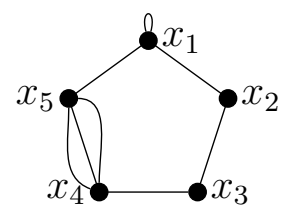

Note that the above graph is sometimes called a pseudo-graph or multi-graph. We will not consider graphs of this type.

Convention 1.2. Throughout these notes, we will assume that $G$ is a finite simple graph, so we will simply call $G$ a graph, and drop the adjectives finite and simple.

We can study graphs using monomial ideals in a suitable polynomial ring. Suppose we are given a graph $G=(V(G), E(G))$ where $V(G)=\left\{x_{1}, \ldots, x_{n}\right\}$. We identify the vertices of the graph with the variables in the polynomial ring $R=k\left[x_{1}, \ldots, x_{n}\right]$. Here, $k$ is some fixed field. (The results of this section are independent of the characteristic of $k$; however, see Example 2.2 for a result that depends upon $\operatorname{char}(k)$.)

The graph $G$ is then used to construct two monomial ideals:

Definition 1.3. Let $G=(V(G), E(G))$ be a graph. The edge ideal associated to $G$ is the monomial ideal

$$
I(G)=\left\langle x_{i} x_{j} \mid\left\{x_{i}, x_{j}\right\} \in E(G)\right\rangle \subseteq R=k\left[x_{1}, \ldots, x_{n}\right] .
$$

The cover ideal is the monomial ideal

$$
J(G)=\bigcap_{\left\{x_{i}, x_{j}\right\} \in E(G)}\left\langle x_{i}, x_{j}\right\rangle \subseteq R=k\left[x_{1}, \ldots, x_{n}\right] .
$$


Example 1.4. Let $G$ be as in Example 1.1. The edge and cover ideals are, respectively:

$$
\begin{aligned}
I(G) & =\left\langle x_{1} x_{2}, x_{2} x_{3}, x_{3} x_{4}, x_{4} x_{5}, x_{5} x_{1}\right\rangle \\
J(G) & =\left\langle x_{1}, x_{2}\right\rangle \cap\left\langle x_{2}, x_{3}\right\rangle \cap\left\langle x_{3}, x_{4}\right\rangle \cap\left\langle x_{4}, x_{5}\right\rangle \cap\left\langle x_{1}, x_{5}\right\rangle \\
& =\left\langle x_{1} x_{3} x_{4}, x_{1} x_{3} x_{5}, x_{1} x_{2} x_{4}, x_{2} x_{4} x_{5}, x_{2} x_{3} x_{5}\right\rangle .
\end{aligned}
$$

Remark 1.5. The term edge ideal originated in a paper of Villarreal [63]. The ideal $J(G)$ is called the cover ideal because its minimal generators correspond to the minimal vertex covers of the graph $G$. We will make this statement precise later in this section.

Observe that we can reverse the construction of Definition 1.3 in the following sense. If $I$ is any quadratic square-free monomial ideal in $R=k\left[x_{1}, \ldots, x_{n}\right]$ of the form $I=$ $\left\langle x_{1,1} x_{1,2}, \ldots, x_{s, 1} x_{s, 2}\right\rangle$ we make the following association:

$$
I \mapsto G=\left(\left\{x_{1}, \ldots, x_{n}\right\},\left\{\left\{x_{1,1}, x_{1,2}\right\}, \ldots,\left\{x_{s, 1}, x_{s, 2}\right\}\right\}\right) .
$$

Similarly, if we are given any square-free unmixed height two monomial ideal, i.e., $J=$ $\bigcap_{i=1}^{t}\left\langle x_{i, 1}, x_{i, 2}\right\rangle$, we identify $J$ with the graph:

$$
J \mapsto G=\left(\left\{x_{1}, \ldots, x_{n}\right\},\left\{\left\{x_{i, 1}, x_{i, 2}\right\} \mid i=1, \ldots, t\right\}\right) .
$$

So, the study of edge and cover ideals hopes to exploit these two one-to-one correspondences:

$$
\left\{\begin{array}{c}
\text { EDGE IDEALS } \\
\text { (quadratic square-free } \\
\text { monomial ideals) }
\end{array}\right\} \stackrel{1-1}{\longleftrightarrow}\left\{\begin{array}{c}
\text { FINITE } \\
\text { SIMPLE } \\
\text { GRAPHS }
\end{array}\right\} \stackrel{1-1}{\longleftrightarrow}\left\{\begin{array}{c}
\text { COVER IDEALS } \\
\text { (height two unmixed } \\
\text { square-free monomial } \\
\text { ideals })
\end{array}\right\} .
$$

Remark 1.6. We have simplified this discussion slightly since one must take into account isolated vertices, or variables of $R$ that do not appear in $I$ or $J$. We gloss over this technicality here to simplify our discussion.

Given these two one-to-one correspondences, it makes sense to ask:

Question 1.7. How do the invariants of finite simple graphs relate to the invariants of the edge and cover ideals, and vice versa?

By answering this broad question about the dictionary between two fields, i.e., graph theory and commutative algebra, we may be able to import results from graph theory to help prove algebraic results, and at the same time, export algebraic results to prove graph theory results.

1.2. Colouring Graphs: an illustration of the dictionary. To give you a hint of the connection between graph theory and commutative algebra, we will examine the problem of colouring a graph. 
Definition 1.8. A colouring ${ }^{1}$ of a graph $G$ is an assignment of a colour to each vertex so that adjacent vertices, i.e., vertices joined by an edge receive different colours. The chromatic number of a graph $G$, denoted $\chi(G)$, is the minimum number of colours needed to colour $G$.

To illustrate this definition, we first introduce two families of graphs that will frequently appear within these notes.

Definition 1.9. The clique of size $n$ with $n \geq 2$, denoted $K_{n}$, is the graph with vertex set $V(G)=\left\{x_{1}, \ldots, x_{n}\right\}$ and edge set $E(G)=\left\{\left\{x_{i}, x_{j}\right\} \mid 1 \leq i<j \leq n\right\}$. We sometimes view an isolated vertex (a vertex with no edges) as a clique of size 1 , and denote it by $K_{1}$.

The cycle of size $n$ with $n \geq 3$, denoted $C_{n}$, is the graph with vertex set $V(G)=$ $\left\{x_{1}, \ldots, x_{n}\right\}$ and edge set $E(G)=\left\{\left\{x_{1}, x_{2}\right\},\left\{x_{2}, x_{3}\right\},\left\{x_{3}, x_{4}\right\}, \ldots,\left\{x_{n-1}, x_{n}\right\},\left\{x_{n}, x_{1}\right\}\right\}$.

Example 1.10. The graphs below are $K_{3}, K_{4}$, and $K_{5}$ :


The graphs below are $C_{3}, C_{4}$, and $C_{5}$ :
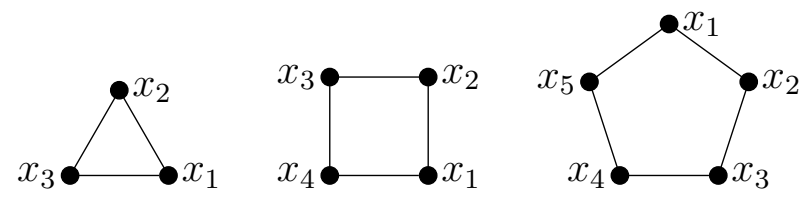

Example 1.11. The chromatic number of $C_{5}$ is three since we can colour it as follows:



where $\mathrm{R}$ represents red, $\mathrm{G}$ represent green, and B represents blue.

For both families of graphs, we can easily compute $\chi(G)$ :

Lemma 1.12. If $G=K_{n}$, then $\chi\left(K_{n}\right)=n$. If $G=C_{n}$, then

$$
\chi\left(C_{n}\right)= \begin{cases}2 & \text { if } n \text { is even } \\ 3 & \text { if } n \text { is odd. }\end{cases}
$$

Proof. (Exercise)

\footnotetext{
${ }^{1}$ I'm using the British-Canadian spelling of colouring, but if you prefer, you can call it a coloring. To be consistent, I'll also use neighbour.
} 
Application 1.13. Colouring is a core topic in graph theory; every introductory textbook on graph theory will devote at least one chapter to the topic. It has many practical applications, including the problem of scheduling. To see the connection, suppose we want to schedule a set of exams. Represent each exam by a vertex, and join two vertices if there is a student who must write both exams. For example, suppose we end up with the graph:

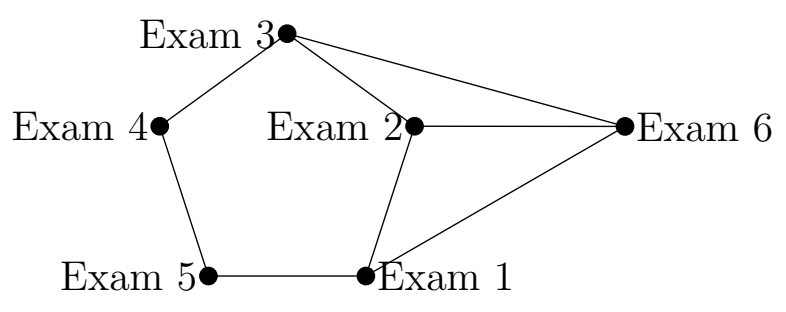

We can colour this graph with three colours (e.g., colour Exam 1 and Exam 3 the same, Exams 5 and 6 are coloured the same, and Exams 2 and 4 are coloured the same). Exams with the same colour can be scheduled at the same time since a student can only take one exam of each colour. The chromatic number of this graph is the smallest number of distinct time slots needed to offer all exams.

Definition 1.14. A subset $W \subseteq V(G)$ is called an independent set if no edge of $G$ has both endpoints in $W$. A maximal independent set is an independent set that is maximal under inclusion.

We make an important observation about a colouring of a graph. Suppose that $G$ has been given a colouring. Then all the vertices of the same colour must form an independent set. This observation leads to the following lemma:

Lemma 1.15. Let $G$ be a graph. Then $\chi(G) \leq d$ if and only if $V(G)=C_{1} \cup \cdots \cup C_{d}$ can be partitioned into $d$ independent sets.

Proof. (Exercise)

There is a concept dual to an independent set:

Definition 1.16. A subset $W \subseteq V(G)$ is a vertex cover if $W \cap e \neq \emptyset$ for all $e \in E(G)$. A vertex cover $W$ is a minimal vertex cover if no proper subset of $W$ is a vertex cover.

From the definitions, one can easily prove the following statement:

Lemma 1.17. A subset $Y \subseteq V(G)$ is an independent set if and only if $V(G) \backslash Y$ is a vertex cover. In particular, $Y$ is a maximal independent set if and only if $V(G) \backslash Y$ is a minimal vertex cover.

Proof. (Exercise) 
Example 1.18. Consider the graph $C_{5}$ of Example 1.1. In this graph, $Y=\left\{x_{2}, x_{5}\right\}$ is an independent set. The complement of $Y$ is the set $V(G) \backslash Y=\left\{x_{1}, x_{3}, x_{4}\right\}$. This set is a vertex cover because every edge of $C_{5}$ has at least one of its endpoints in $\left\{x_{1}, x_{3}, x_{4}\right\}$.

We will now describe how the invariant $\chi(G)$ arises in an algebraic context.

Notation 1.19. If $W \subseteq V(G)$, then

$$
x_{W}:=\prod_{x_{i} \in W} x_{i}
$$

We first require a lemma that justifies the name cover ideal:

Lemma 1.20. Let $G$ be a graph with cover ideal $J(G)$. Then

$$
\left.J(G)=\left\langle x_{W}\right| W \subseteq V(G) \text { is a minimal vertex cover of } G\right\rangle .
$$

Proof. Let $L=\left\langle x_{W}\right| W \subseteq V(G)$ is a minimal vertex cover of $\left.G\right\rangle$.

Let $x_{W}$ be a generator of $L$ with $W$ a minimal vertex cover. Then, for every edge $e=\left\{x_{i}, x_{j}\right\} \in E(G)$, we have $W \cap e \neq \emptyset$. So, either $x_{i} \in W$ or $x_{j} \in W$. Consequently, either $x_{i} \mid x_{W}$ or $x_{j} \mid x_{W}$, whence $x_{W} \in\left\langle x_{i}, x_{j}\right\rangle$. Since $e$ is arbitrary, we have

$$
x_{W} \in \bigcap_{\left\{x_{i}, x_{j}\right\} \in E(G)}\left\langle x_{i}, x_{j}\right\rangle=J(G) .
$$

Conversely, let $m \in J(G)$ be any minimal generator. Note that $m$ must be squarefree since $J(G)$ is the intersection of finitely many square-free monomial ideals. So $m=$ $x_{i_{1}} \cdots x_{i_{r}}$. Let $W=\left\{x_{i_{1}}, \ldots, x_{i_{r}}\right\}$. Since $m \in\left\langle x_{i}, x_{j}\right\rangle$ for each each $\left\{x_{i}, x_{j}\right\} \in E(G)$ either $x_{i} \mid m$ or $x_{j} \mid m$, and thus, $x_{i} \in W$ or $x_{j} \in W$. Thus $W$ is a vertex cover. Let $W^{\prime} \subseteq W$ be a minimal vertex cover. Because $x_{W^{\prime}} \in L$ and $x_{W^{\prime}}$ divides $m=x_{W}$, we have $m \in L$.

Theorem 1.21 ([25, Theorem 3.2]). Let $G$ be a graph with $V(G)=\left\{x_{1}, \ldots, x_{n}\right\}$. If $J(G)$ is the cover ideal, then

$$
\chi(G)=\min \left\{d \mid x_{V(G)}^{d-1}=\left(x_{1} \cdots x_{n}\right)^{d-1} \in J(G)^{d}\right\} .
$$

Proof. Let $J=J(G)$. We first want to show that if $\chi(G)=d$, then $\left(x_{1} \cdots x_{n}\right)^{d-1} \in J^{d}$.

Because $\chi(G)=d$, we have a partition $V_{G}=C_{1} \cup \cdots \cup C_{d}$ into independent sets (see Lemma 1.15). Because each $C_{i}$ is an independent set, the set $W_{i}=V(G) \backslash C_{i}$ is vertex cover, and thus $x_{W_{i}} \in J$. So, $x_{W_{1}} \cdots x_{W_{d}} \in J^{d}$. Each $x_{i} \in V(G)$ is in exactly one $C_{i}$, so each $x_{i}$ is in exactly $d-1$ of the $W_{j}$. Thus $x_{W_{1}} \cdots x_{W_{d}}=\left(x_{1} \cdots x_{n}\right)^{d-1} \in J^{d}$. This shows

$$
\chi(G) \geq \min \left\{d \mid x_{V(G)}^{d-1}=\left(x_{1} \cdots x_{n}\right)^{d-1} \in J^{d}\right\} .
$$

Conversely, let $\left(x_{1} \cdots x_{n}\right)^{d-1} \in J^{d}$. So, we can find $d$ minimal vertex covers $W_{1}, \ldots, W_{d}$ such that

$$
\left(x_{1} \cdots x_{n}\right)^{d-1}=x_{W_{1}} \cdots x_{W_{d}} M \in J^{d}
$$


where $M$ is some monomial (possibly $M=1$ ). Set

$$
\begin{aligned}
C_{1} & =V(G) \backslash W_{1} \\
C_{2} & =\left(V(G) \backslash W_{2}\right) \backslash C_{1} \\
C_{3} & =\left(V(G) \backslash W_{3}\right) \backslash\left(C_{1} \cup C_{2}\right) \\
& \vdots \\
C_{d} & =\left(V(G) \backslash W_{d}\right) \backslash\left(C_{1} \cup \cdots \cup C_{d-1}\right) .
\end{aligned}
$$

Then $C_{1} \cup \cdots \cup C_{d}$ is a colouring of $G$. To see this, first note that each $C_{i}$ is an independent subset because it is a subset of $V(G) \backslash W_{i}$. By construction, for each $i \neq j$, we have $C_{i} \cap C_{j}=\emptyset$. Finally, for any $x_{j} \in V(G)$, there is at least one $W_{i}$ such that $x_{j} \notin W_{i}$. Indeed, if $x_{j} \in W_{i}$ for all $i$, then $x_{j}^{d} \mid x_{W_{1}} \cdots x_{W_{d}} M=\left(x_{1} \cdots x_{n}\right)^{d-1}$, a contradiction. So, $x_{j} \in C_{i}=V(G) \backslash W_{i}$ or $x_{j} \in C_{1} \cup C_{2} \cup \cdots \cup C_{i-1}$.

Thus $\chi(G) \leq \min \left\{d \mid x_{V(G)}^{d-1}=\left(x_{1} \cdots x_{n}\right)^{d-1} \in J^{d}\right\}$, as desired.

Example 1.22. Let $G=C_{5}$. Then a colouring of $G$ is given by

$$
V(G)=\underbrace{\left\{x_{1}, x_{3}\right\}}_{\text {Red }} \cup \underbrace{\left\{x_{2}, x_{4}\right\}}_{\text {Blue }} \cup \underbrace{\left\{x_{5}\right\}}_{\text {Green }} .
$$

Since $\left\{x_{1}, x_{3}\right\},\left\{x_{2}, x_{4}\right\}$, and $\left\{x_{5}\right\}$ are independent sets, the sets $\left\{x_{2}, x_{4}, x_{5}\right\},\left\{x_{1}, x_{3}, x_{5}\right\}$ and $\left\{x_{1}, x_{2}, x_{3}, x_{4}\right\}$ are vertex covers of $G$. So $x_{2} x_{4} x_{5}, x_{1} x_{3} x_{5}$, and $x_{1} x_{2} x_{3} x_{4} \in J(G)$. It follows that

$$
\left(x_{2} x_{4} x_{5}\right)\left(x_{1} x_{3} x_{5}\right)\left(x_{1} x_{2} x_{3} x_{4}\right)=\left(x_{1} x_{2} x_{3} x_{4} x_{5}\right)^{2} \in J(G)^{3} .
$$

Remark 1.23. Theorem 1.21 allows us to compute $\chi(G)$ without finding an explicit colouring of $G$. In the Macaulay2 [31] package EdgeIdeals [29], this formula is used to find the chromatic number. Although we have a simple algorithm for finding the chromatic number, the bottleneck in the computation is finding $J(G)$. As proved in Lemma 1.20 , finding the generators of $J(G)$ is equivalent to finding all the vertex covers of $G$. However, this is an NP problem!

In graph theory, there is also a notion of a $b$-fold chromatic number which we can also compute algebraically.

Definition 1.24. A $b$-fold colouring of a graph $G$ is an assignment to each vertex a set of $b$ distinct colours such that adjacent vertices receive disjoint sets of colours. The $b$-fold chromatic number of $G$, denoted $\chi_{b}(G)$, is the minimal number of colours needed in a $b$-fold colouring of $G$. 
Example 1.25. The 2-fold chromatic number of $C_{5}$ is five since we can colour it as follows:

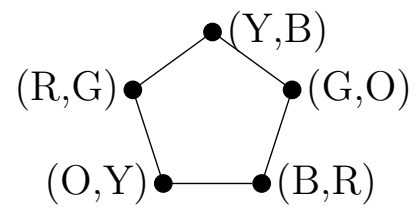

where $\mathrm{R}$ represents red, $\mathrm{G}$ represent green, $\mathrm{B}$ represents blue, $\mathrm{O}$ represents orange, and Y represents yellow.

When $b=1$, then $\chi_{b}(G)=\chi(G)$. By adapting the proof of Theorem 1.21 , we can derive a much more general result. See the paper [25] for a proof.

Theorem 1.26. Let $G$ be a graph with $V(G)=\left\{x_{1}, \ldots, x_{n}\right\}$. If $J(G)$ is the cover ideal, then

$$
\chi_{b}(G)=\min \left\{d \mid x_{V(G)}^{d-b}=\left(x_{1} \cdots x_{n}\right)^{d-b} \in J(G)^{d}\right\} .
$$

Before we turn to the next section, we will end with an open question. The fractional chromatic number of a graph $G$, denoted $\chi_{f}(G)$, is defined to be

$$
\chi_{f}(G)=\lim _{b \rightarrow \infty} \frac{\chi_{b}(G)}{b} .
$$

Then it is known that there exists some integer $b$ such that $\chi_{f}(G)=\frac{\chi_{b}(G)}{b}$. Since we can compute $\chi_{b}(G)$, it is natural to ask:

Question 1.27. Can we compute $\chi_{f}(G)$ algebraically?

If you are interested in the fractional chromatic number, see the book of Scheinermann and Ullman $[55]^{2}$

1.3. Associated Simplicial Complexes. Because $I(G)$ and $J(G)$ are square-free monomial ideals, we can use the Stanley-Reisner construction ${ }^{3}$ to associate to each ideal a simplicial complex. These simplicial complexes are sometimes useful in our study of the invariants of $G$ using the algebraic properties of $I(G)$ and $J(G)$. We quickly discuss the connection.

Definition 1.28. A simplicial complex on $V=\left\{x_{1}, \ldots, x_{n}\right\}$ is a subset $\Delta$ of the power set of $V$ such that

(i) if $F \in \Delta$, and $G \subseteq F$, then $G \in \Delta$.

(ii) $\left\{x_{i}\right\} \in \Delta$ for all $i$.

One can then associate to $\Delta$ a square-free monomial ideal in the ring $R=k\left[x_{1}, \ldots, x_{n}\right]$ :

\footnotetext{
${ }^{2}$ Although the book is out-of-print, you can have a free electronic copy if you send the authors a postcard; see http://www.ams.jhu.edu/ ers/fgt/ for details.

${ }^{3}$ For a refresher on Stanley-Reisner rings, see either Stanley [58] or Bruns and Herzog [4].
} 
Definition 1.29. The Stanley-Reisner ideal associated to a simplicial complex $\Delta$ is the square-free monomial ideal

$$
I_{\Delta}=\left\langle x_{W} \mid W \notin \Delta\right\rangle .
$$

Notice that this operation can be reversed. That is, if $I$ is a square-free monomial ideal in $R$, we can associate to $I$ the simplicial complex

$$
\Delta(I)=\left\{W \subseteq V \mid x_{W} \notin I \text { and } x_{W} \text { square-free }\right\} .
$$

Using some well-known results from Stanley-Reisner theory, we can see how some of the invariants of $G$ are encoded into $I(G)$ and $J(G)$.

Definition 1.30. Let $G$ be a graph. The independence complex of $G$ is the simplicial complex defined by

$$
\Delta(G)=\{W \subseteq V(G) \mid W \text { is an independent set }\} .
$$

The following lemma is then a consequence of the definitions:

Lemma 1.31. Let $G$ a graph with associated simplicial complex $\Delta(G)$. Then

$$
I(G)=I_{\Delta(G)} .
$$

Proof. Let $\Delta$ be the simplicial complex associated to $I(G)$. Then

$$
\begin{aligned}
\Delta & =\left\{W \subseteq V(G) \mid x_{W} \notin I(G)\right\} \\
& =\left\{W \subseteq V(G) \mid \text { for each }\left\{x_{i}, x_{j}\right\} \in E(G), x_{i} x_{j} \nmid x_{W}\right\} \\
& =\{W \subseteq V(G) \mid \text { no subset of } W \text { is an edge }\}=\Delta(G) .
\end{aligned}
$$

Definition 1.32. For a graph $G$, let

$$
\alpha(G)=\max \{|W| \mid W \text { is a maximal independent set }\} .
$$

Recall that elements of a simplicial complex $\Delta$ are called faces, and that if $F \in \Delta$, then $\operatorname{dim} F=|F|-1$. Furthermore, we have $\operatorname{dim} \Delta=\max _{F \in \Delta}\{\operatorname{dim} F\}$. Putting together some of the above pieces gives us:

Theorem 1.33. For a graph $G$,

$$
\operatorname{dim} R / I(G)=\alpha(G) .
$$

Proof. For any simplicial complex $\Delta$, we have $\operatorname{dim} R / I_{\Delta}=\operatorname{dim} \Delta+1$ (see, for example, Theorem 1.3 in Chapter 2 of [58]). So,

$$
\operatorname{dim} R / I(G)=\operatorname{dim} R / I_{\Delta(G)}=\operatorname{dim} \Delta(G)+1=\alpha(G)-1+1=\alpha(G) .
$$


For any simplicial complex $\Delta$, the primary decomposition of $I_{\Delta}$ can be described in terms of the facets of $\Delta$. Recall that $F \in \Delta$ is a facet if $F$ is a face that is maximal under inclusion. For each facet $F$, let

$$
P_{F}=\left\langle x_{i} \mid x_{i} \notin F\right\rangle \text {. }
$$

Theorem 1.34. Let $\Delta$ be a simplicial complex with facets $F_{1}, \ldots, F_{t}$. Then

$$
I_{\Delta}=P_{F_{1}} \cap P_{F_{2}} \cap \cdots \cap P_{F_{t}} .
$$

Proof. For one proof, see [36, Lemma 1.5.4].

We now apply the above result to the simplicial complex $\Delta(G)$. The facets of $\Delta(G)$ are the maximal independent sets of $G$. So, if $F \in \Delta(G)$ is a facet,

$$
P_{F}=\left\langle x_{i} \mid x_{i} \notin F\right\rangle=\left\langle x_{i} \mid x_{i} \in V(G) \backslash F\right\rangle \text {. }
$$

But $V(G) \backslash F$ will be a minimal vertex cover of $G$. Hence, we have

Corollary 1.35. Let $W_{1}, \ldots, W_{t}$ be the minimal vertex covers of $G$, and set $\left\langle W_{i}\right\rangle=$ $\left\langle x_{j} \mid x_{j} \in W_{i}\right\rangle$. Then

$$
I(G)=\left\langle W_{1}\right\rangle \cap \cdots \cap\left\langle W_{t}\right\rangle .
$$

Example 1.36. Consider again the graph $C_{5}$. Then

$$
I\left(C_{5}\right)=\left\langle x_{1}, x_{3}, x_{4}\right\rangle \cap\left\langle x_{1}, x_{3}, x_{5}\right\rangle \cap\left\langle x_{2}, x_{4}, x_{5}\right\rangle \cap\left\langle x_{2}, x_{3}, x_{5}\right\rangle \cap\left\langle x_{1}, x_{2}, x_{4}\right\rangle .
$$

As a consequence of Corollary 1.35, we get an immediate connection between the ideals $I(G)$ and $J(G)$. First, we recall the following important notion:

Definition 1.37. Let $I$ be a square-free monomial ideal with primary decomposition

$$
I=\left\langle x_{1,1}, x_{1,2}, \ldots, x_{1, s_{1}}\right\rangle \cap\left\langle x_{2,1}, x_{2,2}, \ldots, x_{2, s_{2}}\right\rangle \cap \cdots \cap\left\langle x_{t, 1}, x_{1,2}, \ldots, x_{t, s_{t}}\right\rangle .
$$

The Alexander dual of $I$, denoted $I^{\vee}$, is the square-free monomial ideal

$$
I^{\vee}=\left\langle x_{1,1} x_{1,2} \cdots x_{1, s_{1}}, x_{2,1} x_{2,2} \cdots x_{2, s_{2}}, \ldots, x_{t, 1} x_{t, 2} \cdots x_{t, s_{t}}\right\rangle .
$$

Corollary 1.38. Let $G$ be a graph. Then $I(G)^{\vee}=J(G)$.

Example 1.39. In the previous example, we showed that

$$
I\left(C_{5}\right)=\left\langle x_{1}, x_{3}, x_{4}\right\rangle \cap\left\langle x_{1}, x_{3}, x_{5}\right\rangle \cap\left\langle x_{2}, x_{4}, x_{5}\right\rangle \cap\left\langle x_{2}, x_{3}, x_{5}\right\rangle \cap\left\langle x_{1}, x_{2}, x_{4}\right\rangle .
$$

So $I\left(C_{5}\right)^{\vee}=J(G)=\left\langle x_{1} x_{3} x_{4}, x_{1} x_{3} x_{5}, x_{2} x_{4} x_{5}, x_{2} x_{3} x_{5}, x_{1} x_{2} x_{4}\right\rangle$. 
1.4. Additional constructions. In this section, we have seen two ways to associate to a graph $G$ a monomial ideal. However, these are not the only such constructions. We leave off with two additional constructions and some references.

Construction 1.40 (Path Ideals). A path of length $t$ in a graph $G$ is a set of $t$ distinct vertices $\left\{x_{i_{1}}, \ldots, x_{i_{t}}\right\}$ such that $\left\{x_{i_{j}}, x_{i_{j+1}}\right\} \in E(G)$ for $j=1, \ldots, t-1$. The path ideal of length of $t$ of $G$, denoted $I_{t}(G)$ is the monomial ideal

$$
\left.I_{t}(G)=\left\langle x_{i_{1}} \cdots x_{i_{t}}\right|\left\{x_{i_{1}}, \ldots, x_{i_{t}}\right\} \text { is a path of length } t\right\rangle .
$$

Observe that when $t=2$, a path of length 2 is simply an edge, so $I_{2}(G)$ is the edge ideal of $G$. This construction is one way in which we can extend the construction of edge ideals.

Path ideals were first introduced by Conca and De Negri [9]. Further properties have been developed by He and Van Tuyl [35], Bouchat, Hà, and O'Keefe [3], and Alilooee and Faridi [1].

Construction 1.41 (Edge and cover ideals of hypergraphs). Our second construction works with a generalization of graphs. Finite simple graphs can be viewed as a special case of a hypergraph. We first recall this definition. Let $\mathcal{X}=\left\{x_{1}, \ldots, x_{n}\right\}$ be a finite set, and let $\mathcal{E}=\left\{E_{1}, \ldots, E_{s}\right\}$ be a family of distinct subsets of $\mathcal{X}$. The pair $\mathcal{H}=(\mathcal{X}, \mathcal{E})$ is called a hypergraph if $E_{i} \neq \emptyset$ for each $i$. The elements of $\mathcal{X}$ are called the vertices, while the elements of $\mathcal{E}$ are called the edges of $\mathcal{H}$. A hypergraph $\mathcal{H}$ is simple if: (1) $\mathcal{H}$ has no loops, i.e., $|E| \geq 2$ for all $E \in \mathcal{E}$, and $(2) \mathcal{H}$ has no multiple edges, i.e., whenever $E_{i}, E_{j} \in \mathcal{E}$ and $E_{i} \subseteq E_{j}$, then $i=j$. A simple hypergraph is sometimes called a clutter. Notice that a hypergraph generalizes the classical notion of a graph; a graph is a hypergraph for which every $E \in \mathcal{E}$ has cardinality two.

We can then extend the construction of edge and cover ideals to hypergraphs. The edge ideal of $\mathcal{H}$ is the ideal

$$
I(\mathcal{H})=\left\langle x_{E}=\prod_{x \in E} x \mid E \in \mathcal{E}\right\rangle \subseteq R=k\left[x_{1}, \ldots, x_{n}\right] .
$$

The cover ideal of $\mathcal{H}$ is then defined by:

$$
J(\mathcal{H})=\bigcap_{E \in \mathcal{E}}\langle x \mid x \in E\rangle .
$$

The advantage of this construction is that it gives us a one-to-one correspondence between all square-free monomial ideals and all hypergraphs. (Again, I'm glossing over some details about the isolated vertices.) Properties of these ideals have been studied by Emtander [19], Francisco, Hà, and Van Tuyl [25], Hà and Van Tuyl [32] and Morey, Reyes, and Villarreal [51], among others. These ideals have also been called facet ideals; see, for example, Faridi [21].

These constructions are simply the tip of the iceberg. One can make edge rings (see Villarreal [64]) or binomial edge ideals (see Herzog, et al. [37]). Each construction has its own advantage. We end with a very open ended question: 
Question 1.42. Find a new way to associate an algebraic object to a graph. What results can you prove with your correspondence?

\section{Splitting monomial ideals And FröBerg's Theorem}

Let $I$ be a homogeneous ideal in $R=k\left[x_{1}, \ldots, x_{n}\right]$. Suppose that the graded minimal free resolution ${ }^{4}$ of $I$ is given by

$$
0 \rightarrow \bigoplus_{j} R(-j)^{\beta_{l, j}(I)} \rightarrow \cdots \rightarrow \bigoplus_{j} R(-j)^{\beta_{1, j}(I)} \rightarrow \bigoplus_{j} R(-j)^{\beta_{0, j}(I)} \rightarrow I \rightarrow 0 .
$$

Here, $l \leq n$, and $\beta_{i, j}(I)$ is the $(i, j)$ th graded Betti number of $I$. We also let $R(-j)$ denote the polynomial ring shifted in degree $j$.

An important topic in commutative algebra is the study of graded Betti numbers of an ideal. Since all monomial ideals are homogeneous, it makes sense to ask about the Betti numbers of edge and cover ideals. In fact, approaching this topic, we can hope:

Dream 2.1. Describe the graded Betti numbers of $I(G)$ and $J(G)$ using only the properties of the graph $G$.

Unfortunately, we have to call this goal a dream; in reality there exist graphs $G$ where the graded Betti numbers of $I(G)$ depend upon the characteristic of the field $k$ used in the definition of $R=k\left[x_{1}, \ldots, x_{n}\right]$.

Example 2.2. Katzman [43] studied how the characteristic of the field affects the graded Betti numbers of $I(G)$. In particular, he showed that the graph $G$ with vertex set $V(G)=$ $\left\{x_{1}, x_{2}, \ldots, x_{11}\right\}$ and edge set

$$
\begin{aligned}
E(G)= & \left\{x_{1} x_{2}, x_{1} x_{6}, x_{1} x_{7}, x_{1} x_{9}, x_{2} x_{6}, x_{2} x_{8}, x_{2} x_{10}, x_{3} x_{4}, x_{3} x_{5}, x_{3} x_{7}, x_{3} x_{10}, x_{4} x_{5},\right. \\
& \left.x_{4} x_{6}, x_{4} x_{11}, x_{5} x_{8}, x_{5} x_{9}, x_{6} x_{11}, x_{7} x_{9}, x_{7} x_{10}, x_{8} x_{9}, x_{8} x_{10}, x_{8} x_{11}, x_{10} x_{11}\right\} .
\end{aligned}
$$

has the property that the Betti numbers of $I(G)$ when $\operatorname{char}(k)=2$ do not agree with the Betti numbers of $I(G)$ when $\operatorname{char}(k) \neq 2$. Moreover, this graph is the smallest such example, where by smallest we mean that no graph on 10 or less vertices has this feature. Also see the paper of Dalili and Kummini [13] which also studies how the graded Betti numbers of an edge ideal depend upon the characteristic of the field.

Remark 2.3. If you are familiar with Hochster's Formula (see, for example, [49, Corollary 5.12]), then it is well known that the graded Betti numbers of a square-free monomial ideal may depend upon the characteristic of the field. Hochster's Formula shows that the graded Betti numbers of an arbitrary monomial ideal can be computed using reduced simplicial homology, which requires information about the field $k$. However, there are many families of monomial ideals where the Betti numbers are independent of the field; to ask if this fact also applies to edge ideals is a legitimate question that turns out to be false as shown in the previous example.

\footnotetext{
${ }^{4}$ If you are not familiar with this notion, see Peeva [54].
} 
As an aside, if we take the cover ideal $J(G)$ of the graph $G$ in Example 2.2, we find an example of a cover ideal whose Betti numbers also depend upon the char $(k)$ (the Betti numbers of $J(G)$ are different if $\operatorname{char}(k)=2$ ). To the best of my knowledge, this is the smallest such example. I will leave this as a question:

Question 2.4. What is the smallest graph $G$ which has the property that the Betti numbers of $J(G)$, the cover ideal, depend upon the characteristic?

Although Dream 2.1 cannot be realized, we can still ask if some special types of resolutions only depend upon $G$ or if some homological invariants encoded into the resolution only depend upon $G$. We shall focus on linear resolutions and the regularity of an ideal.

Definition 2.5. Let $I$ be a homogeneous ideal generated in degree $d$. Then $I$ has a linear resolution if $\beta_{i, i+j}(I)=0$ for all $j \neq d$.

Another important invariant related to an ideal is the notion of regularity.

Definition 2.6. The Castelnuovo-Mumford regularity (or simply, regularity) of an ideal $I$ is

$$
\operatorname{reg}(I)=\max \left\{j-i \mid \beta_{i, j}(I) \neq 0\right\}
$$

These two notions are linked:

Lemma 2.7. Let $I$ be a homogeneous ideal generated in degree $d$. Then $I$ has a linear resolution if and only if $\operatorname{reg}(I)=d$.

Proof. (Exercise)

Example 2.8. Consider the edge ideal of $C_{5}$, i.e., $I\left(C_{5}\right)=\left\langle x_{1} x_{2}, x_{2} x_{3}, x_{3} x_{4}, x_{4} x_{5}, x_{5} x_{1}\right\rangle$. The graded minimal free resolution of $I\left(C_{5}\right)$ is then given by

$$
0 \rightarrow R(-5) \rightarrow R^{5}(-3) \rightarrow R^{5}(-2) \rightarrow I\left(C_{5}\right) \rightarrow 0 .
$$

So, $\beta_{2,5}(I)=1, \beta_{1,3}(I)=5$, and $\beta_{0,2}(I)=5$. Then

$$
\operatorname{reg}\left(I\left(C_{5}\right)\right)=\max \{2-0,3-1,5-2\}=3 .
$$

Because $I\left(C_{5}\right)$ is generated in degree 2, and $\operatorname{reg}\left(I\left(C_{5}\right)\right)>2$, this ideal does not have a linear resolution.

Although Dream 2.1 cannot be answered, perhaps we can answer the following question:

Question 2.9. Which edge ideals have a linear resolution? Or equivalently, which edge ideals have $\operatorname{reg}(I(G))=2$ ?

This question was first answered by Fröberg [30]. To give the answer, we need some terminology from graph theory. 
Definition 2.10. A cycle of length $t$, denoted $\left(x_{1} x_{2} \cdots x_{t} x_{1}\right)$ is a set of vertices of $G$ such that $\left\{x_{1}, x_{2}\right\},\left\{x_{2}, x_{3}\right\}, \ldots,\left\{x_{t}, x_{1}\right\} \in E(G)$. A chord is an edge joining nonadjacent vertices in a cycle. A minimal cycle is a cycle with no chords. A chordal graph is a graph where all the minimal cycles have length three.

Definition 2.11. If $G=(V(G), E(G))$ is a graph, the complementary graph of $G$, denoted $G^{c}$, is the graph $G^{c}=\left(V(G), E\left(G^{c}\right)\right)$ where

$$
E\left(G^{c}\right)=\left\{\left\{x_{i}, x_{j}\right\} \mid\left\{x_{i}, x_{j}\right\} \notin E(G)\right\} .
$$

Example 2.12. Consider the following three graphs:
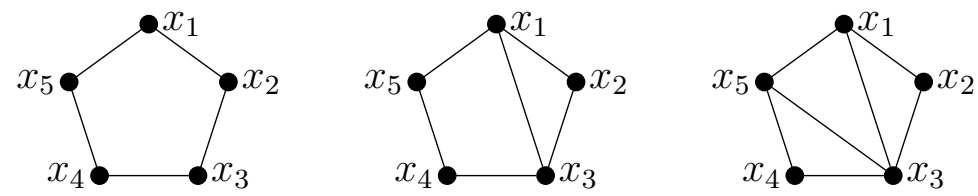

The graph on the left is simply $C_{5}$, the cycle of length 5 . The second graph has a chord between $x_{1}$ and $x_{3}$. Note that $\left(x_{1} x_{2} x_{3} x_{4} x_{5} x_{1}\right)$ is still a cycle of length 5 ; however, it is no longer a minimal cycle. The second graph has two minimal cycles, i.e., $\left(x_{1} x_{2} x_{3} x_{1}\right)$ and $\left(x_{1} x_{3} x_{4} x_{5} x_{1}\right)$. The graph on the right is a chordal graph because all the minimal cycles have length three.

The complements of the above three graphs are, respectively:
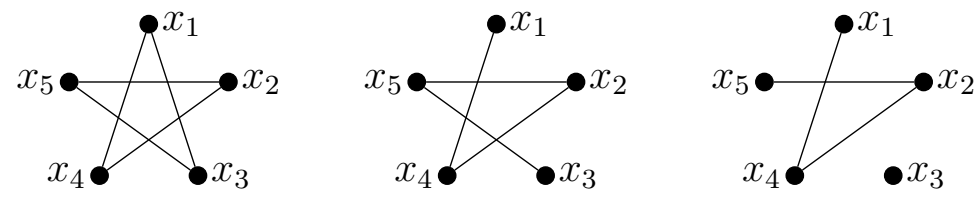

Theorem 2.13 (Fröberg's Theorem [30]). Let $G$ be a graph. Then $I(G)$ has a linear resolution if and only if $G^{c}$ is a chordal graph.

Example 2.14. If $G=C_{5}$, then $G^{c}$ is not a chordal graph, so $I(G)$ will not have a linear resolution (as we already observed).

Fröberg's Theorem is an important result in the study of edge ideals. There are a number of different proofs of this pivotal result. Besides Fröberg's original paper, you can find different proofs in Dochtermann and Engström [15], Eisenbud, et al. [17], Herzog and Hibi [36], and Nevo [52]. We will introduce a technique called splitting that enables us to derive some information about the Betti numbers of ideals. As an application, we will show how to use this technique to prove Fröberg's Theorem.

2.1. Splitting Monomial Ideals. Let $I$ be a monomial ideal, and let $\mathcal{G}(I)=\left\{m_{1}, \ldots, m_{r}\right\}$ denote the set of monomial minimal generators of $I$. Suppose we partition $\mathcal{G}(I)$ into two sets, i.e.,

$$
\mathcal{G}(I)=\left\{m_{1}, \ldots, m_{s}\right\} \cup\left\{m_{s+1}, \ldots, m_{r}\right\}=\mathcal{G}(J) \cup \mathcal{G}(K) .
$$

Let $J=\left\langle m_{1}, \ldots, m_{s}\right\rangle$ and $K=\left\langle m_{s+1}, \ldots, m_{r}\right\rangle$. Note that $I=J+K$. 
We then have a short exact sequence:

$$
0 \rightarrow J \cap K \rightarrow J \oplus K \rightarrow J+K=I \rightarrow 0 .
$$

If we assume that we are given graded minimal free resolutions of $J \cap K, J$, and $K$, then we can use the mapping cone construction $5^{5}$ to build a graded resolution of $I$.

To make this more precise, suppose that we are given the graded minimal free resolutions:

$$
\begin{gathered}
0 \rightarrow F_{l_{1}} \rightarrow F_{l_{1}-1} \rightarrow \cdots \rightarrow F_{1} \rightarrow F_{0} \rightarrow J \cap K \rightarrow 0 \\
0 \rightarrow G_{l_{2}} \rightarrow G_{l_{2}-1} \rightarrow \cdots \rightarrow G_{1} \rightarrow G_{0} \rightarrow J \rightarrow 0
\end{gathered}
$$

and

$$
0 \rightarrow H_{l_{3}} \rightarrow H_{l_{3}-1} \rightarrow \cdots \rightarrow H_{1} \rightarrow H_{0} \rightarrow K \rightarrow 0 .
$$

Note that $F_{i}=\bigoplus_{j} R^{\beta_{i, j}(J \cap K)}(-j)$, and similarly for $G_{i}$ and $H_{i}$. The mapping cone construction then implies that we can construct the following graded resolution from the above three resolutions:

$$
\cdots \rightarrow G_{2} \oplus H_{2} \oplus F_{1} \rightarrow G_{1} \oplus H_{1} \oplus F_{0} \rightarrow G_{0} \oplus H_{0} \rightarrow I \rightarrow 0 .
$$

What is important to notice is that this resolution may or may not be a minimal resolution.

Because the Betti numbers of $I$ can only be smaller than the ones given in the above resolution, we always have the following inequality

$$
\beta_{i, j}(I) \leq \beta_{i, j}(J)+\beta_{i, j}(K)+\beta_{i-1, j}(J \cap K) .
$$

We are interested in when $(\star)$ is an equality. First let us give a name to this situation.

Definition 2.15. We call $I=J+K$ a Betti splitting if for all $i, j \geq 0$,

$$
\beta_{i, j}(I)=\beta_{i, j}(J)+\beta_{i, j}(K)+\beta_{i-1, j}(J \cap K) .
$$

At first glance, we have no evidence that a Betti splitting should even exist. Let us turn to an example.

Example 2.16. We return to our favourite example: $G=C_{5}$. For this case, $I\left(C_{5}\right)=$ $\left\langle x_{1} x_{2}, x_{2} x_{3}, x_{3} x_{4}, x_{4} x_{5}, x_{5} x_{1}\right\rangle$. We partition the generators of $I(G)$ as follows:

$$
J=\left\langle x_{1} x_{2}, x_{5} x_{1}\right\rangle \text { and } K=\left\langle x_{2} x_{3}, x_{3} x_{4}, x_{4} x_{5}\right\rangle .
$$

Then a simple computation will show that $J \cap K=\left\langle x_{1} x_{4} x_{5}, x_{1} x_{2} x_{3}\right\rangle$. We now take the minimal graded free resolutions of $J, K$, and $J \cap K$ :

$$
\begin{gathered}
0 \rightarrow R(-5) \rightarrow R^{2}(-3) \rightarrow J \cap K \rightarrow 0, \\
0 \rightarrow R(-3) \rightarrow R^{2}(-2) \rightarrow J \rightarrow 0,
\end{gathered}
$$

and

$$
0 \rightarrow R^{2}(-3) \rightarrow R^{3}(-2) \rightarrow K \rightarrow 0
$$

\footnotetext{
${ }^{5}$ For more details on this construction, see [54, Chapter 1, Section 27].
} 
The mapping cone construction then gives us a resolution of $I$ :

$$
0 \rightarrow R(-5) \rightarrow R^{2}(-3) \oplus R^{2}(-3) \oplus R(-3) \rightarrow R^{2}(-2) \oplus R^{3}(-2) \rightarrow I \rightarrow 0 .
$$

Note that $R^{2}(-3) \oplus R^{2}(-3) \oplus R(-3)=R^{5}(-3)$ and $R^{2}(-2) \oplus R^{3}(-2)=R^{5}(-2)$. So, the mapping cone construction actually gives the minimal free resolution of $I$, and thus $I=J+K$ is a Betti splitting.

So, Betti splittings exist! Moreover, we get a hint of why they are interesting in the last example. We want to split the ideal $I$ into "smaller" ideals $J, K$, and $J \cap K$, whose resolutions are either easier to compute, or perhaps we know through induction. We can then build the resolution of the "larger" ideal $I$.

What we would therefore like are conditions under which a monomial ideal $I$ has a Betti splitting. Splittings of monomial ideals were first introduced by Eliahou and Kervaire [18]. You are encouraged to look at their criterion for splitting a monomial ideal; we won't reproduce it here because it is somewhat complex and we do not need it. The notion of a splitting was further studied by the C. Francisco, T. Hà, and the author [28]. We will highlight one special case in which monomial ideals can be split.

Theorem 2.17 (Francisco-Hà-Van Tuyl [28]). Let $I \subseteq R=k\left[x_{1}, \ldots, x_{n}\right]$ be a monomial ideal. Fix a variable $x_{i}$, and set

$$
J=\left\langle m \in \mathcal{G}(I)\left|x_{i}\right| m\right\rangle \text { and } K=\left\langle m \in \mathcal{G}(I) \mid x_{i} \nmid m\right\rangle .
$$

(We call this an $x_{i}$-partition of $\mathcal{G}(I)$ ). If $J$ has a linear resolution, then $I=J+K$ is a Betti splitting.

For any graph $G$, if $x \in V(G)$, then the neighbours of $x$ is the set

$$
N(x)=\{y \mid\{x, y\} \in E(G)\} .
$$

We can now describe one way to split the edge ideal $I(G)$ :

Corollary 2.18. Suppose that $G \backslash\{x\}$ (the graph with the vertex $x$ and all adjacent edges removed) is not the graph of isolated vertices. Let $N(x)=\left\{x_{1}, \ldots, x_{t}\right\}$. Then

$$
I(G)=\left\langle x x_{1}, \ldots, x x_{t}\right\rangle+I(G \backslash\{x\})
$$

is a Betti splitting of $I(G)$.

Proof. We have formed an $x$-partition of $\mathcal{G}(I(G))$. Use Theorem 2.17 and the fact that $\left\langle x x_{1}, \ldots, x x_{t}\right\rangle$ has a linear resolution.

Example 2.19. Observe that Example 2.16 is explained by this corollary since $I\left(C_{5}\right)=$ $\left\langle x_{1} x_{2}, x_{1} x_{5}\right\rangle+\left\langle x_{2} x_{3}, x_{3} x_{4}, x_{4} x_{5}\right\rangle$ is an $x_{1}$-splitting of $I\left(C_{5}\right)$.

To make use of Corollary 2.18, we require information about

$$
J \cap K=\left\langle x x_{1}, \ldots, x x_{t}\right\rangle \cap I(G \backslash\{x\}) .
$$


As we shall see, $J \cap K$ is the sum of a collection of edge ideals constructed from $G$. We introduce some notation. Fix a vertex $x \in V(G)$, and let $N(x)=\left\{x_{1}, \ldots, x_{t}\right\}$ be the set of neighbours of $x$. For $i=1, \ldots, t$, set

$$
G_{i}=G \backslash\left(N(x) \cup N\left(x_{i}\right)\right) .
$$

We also let $G_{(x)}$ be the graph with edge set

$$
\{\{u, v\} \in E(G) \mid\{u, v\} \cap N(x) \neq \emptyset, u \neq x \text {, and } v \neq x\} .
$$

Example 2.20. We illustrate this example with $G=C_{5}$. Consider the vertex $x_{1}$. So, $N\left(x_{1}\right)=\left\{x_{2}, x_{5}\right\}$. The graph $G_{1}=G \backslash\left(N\left(x_{1}\right) \cup N\left(x_{2}\right)\right)$ is simply the isolated vertex $x_{4}$, while graph $G_{2}=G \backslash\left(N\left(x_{1}\right) \cup N\left(x_{5}\right)\right)$ is the isolated vertex $x_{3}$. The graph $G_{\left(x_{1}\right)}$ is the graph:

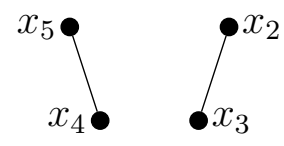

With this notation, we have

Lemma 2.21. Suppose that $G \backslash\{x\}$ is not the graph of isolated vertices. Let $N(x)=$ $\left\{x_{1}, \ldots, x_{t}\right\}$. Then

$$
\left\langle x x_{1}, \ldots, x x_{t}\right\rangle \cap I(G \backslash\{x\})=x I\left(G_{(x)}\right)+x x_{1} I\left(G_{1}\right)+\cdots+x x_{t} I\left(G_{t}\right) .
$$

Proof. See [34].

Example 2.22. Continuing with the above example, the previous lemma thus implies that if $G=C_{5}$ and $x=x_{1}$, then we have

$$
\left\langle x_{1} x_{2}, x_{1} x_{5}\right\rangle \cap\left\langle x_{2} x_{3}, x_{3} x_{4}, x_{4} x_{5}\right\rangle=x_{1}\left\langle x_{2} x_{3}, x_{4} x_{5}\right\rangle+x_{1} x_{2}\langle 0\rangle+x_{1} x_{5}\langle 0\rangle=\left\langle x_{1} x_{2} x_{3}, x_{1} x_{4} x_{5}\right\rangle .
$$

2.2. Proof of Fröberg's Theorem. We will use the machinery of the last section to prove Fröberg's Theorem (Theorem 2.13). Interestingly, once we have set up this machinery, the "tough" part of the proof boils down to proving the following graph theoretic result.

Lemma 2.23. Suppose $G$ is a graph and $x \in V(G)$ is such that $G \backslash\{x\}$ is not the graph of isolated vertices. Then the following are equivalent:

(i) $G^{c}$ is chordal

(ii) (a) $(G \backslash\{x\})^{c}$ is chordal

(b) $G_{(x)}^{c}$ is chordal

(c) $G_{i}$ has no edges.

Proof. Let $N(x)=\left\{x_{1}, \ldots, x_{t}\right\}$.

$(i) \Rightarrow(i i)$. Statement $(a)$ comes from the fact that $(G \backslash\{x\})^{c}=G^{c} \backslash\{x\}$ is an induced subgraph of $G^{c}$, and the chordal property is preserved when passing to induced subgraphs. 
We now show that $(c)$ is true. Suppose that there is an $i$ such that $G_{i}=G \backslash(N(x) \cup$ $\left.N\left(x_{i}\right)\right)$ has an edge, say $\{u, v\}$. By construction, the edges $\{x, u\},\{x, v\},\left\{x_{i}, u\right\}$ and $\left\{x_{i}, v\right\}$ do not belong to $G$. But since $\left\{x, x_{i}\right\}$ and $\{u, v\}$ belong to $G$, in $G^{c}$ we will have the minimal four cycle $\left(x u x_{i} v x\right)$, contradicting the fact that $G^{c}$ is chordal. So $G_{i}$ has no edge.

Finally, we prove $(b)$. The difficulty in proving $(b)$ comes from the fact that $G_{(x)}^{c}$ is not an induced subgraph of $G^{c}$. So, suppose $\left(z_{1} z_{2} \cdots z_{d} z_{1}\right)$ is a minimal cycle in $G_{(x)}^{c}$.

The vertices of $G_{(x)}^{c}$ are the same as $G_{(x)}$, i.e.,

$$
\begin{aligned}
V\left(G_{(x)}^{c}\right)=V\left(G_{(x)}\right) & =N(x) \cup\left\{y \mid\left\{y, x_{i}\right\} \in E(G), x_{i} \in N(x) \text { and } y \notin N(x)\right\} \\
& =N(x) \cup N^{\prime} .
\end{aligned}
$$

We break the proof into four cases depending upon the number of vertices of the cycle $\left(z_{1} \cdots z_{d} z_{1}\right)$ that belong to $N^{\prime}$.

(A) If $\left\{z_{1}, \ldots, z_{d}\right\} \subseteq N(x)$, i.e., no vertices belong to $N^{\prime}$, then the induced graph on $G^{c}$ on these vertices is still a cycle, so $d=3$, since $G^{c}$ is chordal.

$(B)$ If exactly one of $\left\{z_{1}, \ldots, z_{d}\right\}$ is in $N^{\prime}$, then again the induced graph on these vertices is still a cycle in $G^{c}$, so $d=3$.

$(C)$ If exactly two of $\left\{z_{1}, \ldots, z_{d}\right\}$ belong to $N^{\prime}$, say $z_{i}$ and $z_{j}$, then since $z_{i}$ and $z_{i}$ are not adjacent in $G_{(x)},\left\{z_{i}, z_{j}\right\} \in E\left(G_{(x)}^{c}\right)$. Because this cycle is a minimal cycle, this edge must be part of the cycle. So, after relabelling, we can assume $\left\{z_{1}, z_{2}\right\}$ is an edge of the cycle, with both vertices in $N^{\prime}$. In $G^{c}$, the vertex $x$ is adjacent to $z_{1}$ and $z_{2}$, but none of $\left\{z_{3}, \ldots, z_{d}\right\}$. So $\left(z_{1} x z_{2} \cdots z_{d} z_{1}\right)$ is a cycle in $G^{c}$.

There are now two cases. If $\left\{z_{1}, z_{2}\right\} \in E(G)$, then $\left(z_{1} x z_{2} \cdots z_{d} z_{1}\right)$ is a minimal cycle of length $d+1$ in $G^{c}$. Since $G^{c}$ is chordal, $d+1=3$, so $d=2$. But $\left(z_{1} z_{2} z_{1}\right)$ is not a cycle, thus a contradiction. If $\left\{z_{1}, z_{2}\right\} \notin E(G)$, then $\left\{z_{1}, z_{2}\right\}$ is a chord in the cycle $\left(z_{1} x z_{2} \cdots z_{d} z_{1}\right)$ in $G^{c}$. So $\left(z_{1} z_{2} \cdots z_{d} z_{1}\right)$ is minimal cycle in $G^{c}$, whence $d=3$.

$(D)$ If three or more vertices of the cycle belong to $N^{\prime}$, then since none of these vertices of $N^{\prime}$ are adjacent in $G_{(x)}$, these vertices form a clique of size $\geq 3$ in $G_{(x)}^{c}$. A minimal circuit contains a clique only if $d=3$.

We now show $(i i) \Rightarrow(i)$. By $(a)$, if $G^{c}$ has a cycle of length $\geq 4$, then it must pass through the vertex $x$. Let $\left(x z_{1} z_{2} \cdots z_{d} x\right)$ with $d \geq 3$ be this cycle. We break this into two cases: $d \geq 4$ and $d=3$.

If $d \geq 4$, then $\left\{x, z_{2}\right\}, \ldots,\left\{x, z_{d-1}\right\} \notin E\left(G^{c}\right)$ which implies that they all belong to $E(G)$, and thus $\left\{z_{2}, \ldots, z_{d-1}\right\} \subseteq N(x)$. Because the edges $\left\{x, z_{1}\right\}$ and $\left\{x, z_{d}\right\}$ belong to $E\left(G^{c}\right)$, they do not belong to $E(G)$, so $z_{1}, z_{d} \notin N(x)$. In addition, the edges $\left\{z_{1}, z_{d-1}\right\}$ and $\left\{z_{d}, z_{2}\right\}$ are not in $E\left(G^{c}\right)$, so $z_{1}, z_{2} \in N^{\prime}$, where $N^{\prime}$ is the same set introduced in the first half of the proof. So $\left\{z_{1}, z_{d}\right\} \notin E\left(G_{(x)}\right)$, or equivalently, $\left\{z_{1}, z_{d}\right\} \in E\left(G_{(x)}^{c}\right)$. Thus, in the graph $G_{(x)}^{c}$ we have the minimal cycle $\left(z_{1} z_{2} \ldots z_{d} z_{1}\right)$ of length at least four, which contradicts $(b)$. 
Now suppose $d=3$, i.e., suppose $\left(x z_{1} z_{2} z_{3} x\right)$ is a minimal four cycle in $G^{c}$. So $\left\{x, z_{2}\right\}$ and $\left\{z_{1}, z_{3}\right\}$ must be edges in $E(G)$ since they are not edges of $G^{c}$. Note that $z_{1}, z_{3} \notin N(x)$. Because $z_{2} \in N(x)$ and because $G \backslash\left(N(x) \cup N\left(z_{2}\right)\right)$ has no edges, either $z_{1} \in N\left(z_{2}\right)$ or $z_{3} \in N\left(z_{2}\right)$. Indeed, if $z_{1}, z_{3} \notin N\left(z_{2}\right)$, then $\left\{z_{1}, z_{3}\right\}$ would be an edge of $G \backslash\left(N(x) \cup N\left(z_{2}\right)\right)$. So, either $\left\{z_{1}, z_{2}\right\}$ or $\left\{z_{2}, z_{3}\right\}$ are edges of $G$. But this contradicts the fact that both edges $\left\{z_{1}, z_{2}\right\}$ and $\left\{z_{2}, z_{3}\right\}$ belong to $G^{c}$. So $G^{c}$ has no minimal cycle of length $\geq 4$, and thus, $G^{c}$ must be chordal.

We now come to the main result of the section:

Proof. (of Fröberg's Theorem [Theorem 2.13]) We do induction on $|V(G)|$. If $|V(G)| \leq 3$, one simply checks all possible graphs. If $G$ is the graph

$$
G=\left(\left\{x, x_{1}, \ldots, x_{t}\right\},\left\{\left\{x, x_{1}\right\},\left\{x, x_{2}\right\}, \ldots,\left\{x, x_{t}\right\}\right\}\right)
$$

for some $t \geq 1$, then $I(G)=x\left(x_{1}, \ldots, x_{t}\right)$ has a linear resolution and $G^{c}$ is chordal.

So, we can assume that $|V(G)| \geq 4$ and that there is a vertex $x \in V(G)$ such that $G \backslash\{x\}$ is not the graph of isolated vertices. Thus, there is a Betti splitting of $I(G)$, whence

$$
\beta_{i, j}(I(G))=\beta_{i, j}\left(\left(x x_{1}, \ldots, x x_{t}\right)\right)+\beta_{i, j}(I(G \backslash\{x\}))+\beta_{i-1, j}(L)
$$

where $L=x I\left(G_{(x)}\right)+x x_{1} I\left(G_{1}\right)+\cdots+x x_{t} I\left(G_{t}\right)$.

Let us suppose that $I(G)$ has a linear resolution. This implies that $\left(x x_{1}, \ldots, x x_{t}\right)$, $I(G \backslash\{x\})$, and $L$ all have a linear resolution. By induction $(G \backslash\{x\})^{c}=G^{c} \backslash\{x\}$ is chordal. Because $L$ has a linear resolution, we must have $L=x I\left(G_{(x)}\right)$ since $L$ cannot have generators in degree three and four. So, that means $G_{i}$ has no edges for $i=1, \ldots, t$. Finally, since $L=x I\left(G_{(x)}\right)$ has a linear resolution, then so must $I\left(G_{(x)}\right)$, and thus by induction $G_{(x)}^{c}$ is chordal. Now apply Lemma 2.23 to deduce that $G^{c}$ is chordal.

Conversely, suppose $G^{c}$ is chordal. The ideal $\left(x x_{1}, \ldots, x x_{t}\right)$ always has a linear resolution. By Lemma 2.23, $(G \backslash\{x\})^{c}$ is chordal, thus by induction, $I(G \backslash\{x\})$ has a linear resolution. Again, Lemma 2.23 implies that $L=x I\left(G_{(x)}\right)$ with $G_{(x)}^{c}$ chordal. So, by induction, $L$ has linear resolution. Hence, our splitting formula $(\star)$ implies $I(G)$ has a linear resolution.

Remark 2.24. Since the appearance of Fröberg's Theorem, there have been a number of interesting generalizations. Eisenbud, et. al [17] describe how long the resolution will stay linear (sometimes called the $N_{2, p}$ property) in terms of the length of the smallest induced cycle in the complement of $G$ (also see Tutorial Exercise 5.2.3 and 5.2.4 at the end of this paper for more details). Some additional results in this direction were discovered by Fernández-Ramos and Gimenez [23]. Finding a hypergraph version of Fröberg's Theorem appears much more difficult, in part, because the characteristic of the field becomes relevant. See [20, 32, 67] for some known facts about this case.

\subsection{Additional comments and open questions.}


2.3.1. Splitting monomial ideals. We have only scratched the surface when it comes to splitting ideals. As mentioned, this idea first arose in a paper of Eliahou and Kervaire [18. Further properties were developed by Francisco, Hà and Van Tuyl [28].

You should see splitting as a useful tool to deduce results about monomial ideals. For example, T. Hà and myself found a number of results about the edge ideals of graphs and hypergraphs [32, 34]. Francisco [24], Fatabbi [22] and Valla [61] have all used the splitting of monomial ideals to study the ideal of fat points in projective space $\mathbb{P}^{n}$. Hopefully this section has shown you that the technique of splitting a monomial ideal should belong to your toolbox.

There are many questions that can still be asked. In this section, we looked at one way to split $I(G)$. It makes sense to ask:

Question 2.25. Can we find other splittings of the edge ideal $I(G)$ ?

As an example of another answer to this question, T. Hà and myself [34] introduced what we called edge splittings, i.e., finding conditions under which

$$
I(G)=\left\langle x_{i} x_{j}\right\rangle+I(G \backslash e)
$$

is a splitting; here $G \backslash e$ is the graph $G$ with the edge $e=\left\{x_{i}, x_{j}\right\}$ removed. This splitting lead to a nice recursive formula for the graded Betti of chordal graphs [32]. Hibi, Kimura, and Murai where then able to exploit this formula in their study of $f$-vectors (see [39]); this formula was also used by Kimura [45]. There may be other ways to split $I(G)$.

As you may have noticed, we have not said much about the cover ideal $J(G)$ in this section. Of course, we can ask

Question 2.26. Can we find splittings of the cover ideal $J(G)$ ?

I know of only one special case where a splitting of $J(G)$ has been found. C. Francisco, T. Hà, and myself [28] showed that $J(G)$ can be split if $R / I(G)$ is Cohen-Macaulay, and the graph $G$ is bipartite. It would be nice to find other natural ways to split $J(G)$.

2.3.2. Regularity of edge ideals. The regularity of ideals is a fascinating topic. Even in the case of edge ideals, there is much more we would like to know.

As noted, Fröberg's Theorem classifies all the edge ideals which have regularity two. Herzog, Hibi, and Zheng have given a very interesting generalization of this theorem:

Theorem 2.27 ([38]). If $G^{c}$ is chordal, then $I(G)^{s}$ has a linear resolution for all $s \geq 1$. In particular, $\operatorname{reg}\left(I(G)^{s}\right)=2 s$.

Nevo and Peeva [53] have made a conjecture about the regularity of edge ideals, which generalizes this theorem.

Conjecture 2.28. Suppose that $G^{c}$ has no minimal 4-cycles. Then there exists an integer $t$ such that $I(G)^{s}$ has a linear resolution for all $s \geq t$. In particular, $\operatorname{reg}\left(I(G)^{s}\right)=2 s$ for $s \gg 0$. 
Nevo [52] and Hoefel and Whieldon [40] have given some partial evidence for this conjecture. Nevo has shown that if the graph $G$ has the property that $G^{c}$ has no minimal four cycle, and $G$ has no claw, then $I(G)^{2}$ has a linear resolution. A claw is any induced subgraph of the form

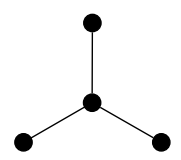

Hoefel and Whieldon have shown that if $G=C_{n}^{c}$, the complement of an $n$-cycle, then $I(G)^{2}$ has a linear resolution, even though $I(G)$ does not.

Finally, if you are interested in learning more about the regularity of edge ideals, the following papers should be part of your reading list: [12, 32, 44, 46, 50, 62, 65, 66, 68,

2.3.3. Simplicial and Cellular Resolutions. We have focused primarily on the case of computing graded Betti numbers (or bounding these invariants, as in the case of regularity). There has also been some interest in describing the structure (e.g., describing the maps) of the minimal free resolutions of edge and cover ideals.

In addition, one would like to determine if there exists a simplicial complex or cell complex that supports a particular resolution. It is beyond the scope of these notes to define these terms, so we point the reader to the textbook of Peeva [54]. We will simply say that one wishes to identify topological objects that encode the structure of the minimal free resolution of a monomial ideal.

To date, most investigations have focused on the structure of the minimal free resolutions of edge ideals. Biermann [2] looked at the resolutions of $I\left(C_{n}^{c}\right)$, i.e., the edge ideal of the complement of cycles; Chen [6] and Horwitz [41] examined the case of ideals with linear resolutions, i.e., ideals of the form $I(G)$ with $G^{c}$ chordal; Corso and Nagel [11, 12] considered the case of Ferrers graph; and Dochtermann and Engström [16] studied the cellular resolutions of co-interval graphs. I do not know of any work on the structure of cover ideals of graphs.

\section{Colouring GRAPHS AND DECOMPOSING COVER IDEALS}

Let $I \subseteq R=k\left[x_{1}, \ldots, x_{n}\right]$ be any ideal. Recall that a prime ideal $P$ is associated to an ideal $I$ if there exists an element $m \in R$ such that $I:\langle m\rangle=P$. The set of associated primes is then the set

$$
\operatorname{Ass}(I)=\{P \mid P \text { is associated to } I\}
$$

In the case that $I$ is a monomial ideal, all the associated prime ideals must also be monomial. Furthermore, the only prime monomial ideals are those of the form $\left\langle x_{i_{1}}, \ldots, x_{i_{r}}\right\rangle$. 
In the case that $I=I(G)$ or $I=J(G)$, we already know the associated primes from Section 1:

$$
\begin{aligned}
\operatorname{Ass}(I(G)) & =\left\{\left\langle x_{i_{1}}, \ldots, x_{i_{t}}\right\rangle \mid\left\{x_{i_{1}}, \ldots, x_{i_{r}}\right\} \text { is a minimal vertex cover }\right\} \\
\operatorname{Ass}(J(G)) & =\left\{\left\langle x_{i}, x_{j}\right\rangle \mid\left\{x_{i}, x_{j}\right\} \in E(G)\right\} .
\end{aligned}
$$

Note that the variables that generate the associated primes of $I(G)$ and $J(G)$ are telling us something about the graph $G$ at the corresponding vertices.

Since we already know $\operatorname{Ass}(I)$ for $I=I(G)$ and $I=J(G)$, we can ask:

Problem 3.1. Suppose $I=I(G)$ or $I=J(G)$. Describe the sets $\operatorname{Ass}\left(I^{s}\right)$ as s varies.

We will focus on the case that $I=J(G)$. For the case of the edge ideal, see the papers of Chen, Morey, and Sung [5] and Martinez-Bernal, Morey, and Villarreal [48].

As a teaser, we already saw in Section 1 that the chromatic number of $G$ was encoded into information about the powers of $J(G)$. We will discover that additional colouring information will be encoded into the powers of $J(G)$.

Before proceeding, we first point out that we will be abusing notation slightly. We shall let $P$ denote both the monomial ideal $\left\langle x_{i_{1}}, \ldots, x_{i_{t}}\right\rangle$ and the subset $\left\{x_{i_{1}}, \ldots, x_{i_{t}}\right\} \subseteq V(G)$. It will be clear from the context whether $P$ is an ideal or a subset of $V(G)$.

In addition, we need the notion of an induced subgraph. Let $P \subseteq V(G)$. The induced graph of $G$ on $P$ is the graph

$$
G_{P}=\left(P, E\left(G_{P}\right)\right)=\left(P,\left\{\left\{x_{i}, x_{j}\right\} \in E(G) \mid\left\{x_{i}, x_{j}\right\} \subseteq P\right\}\right) .
$$

Example 3.2. Consider the graph $G=C_{5}$, and let $P=\left\{x_{2}, x_{3}, x_{5}\right\}$. Then the induced graph $G_{P}$ is the graph:

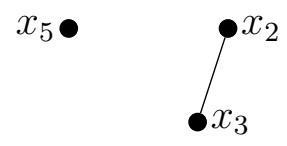

3.1. Powers of cover ideals: associated primes. We begin with a lemma that reduces our problem to determining if the maximal ideal $\left\langle x_{1}, \ldots, x_{n}\right\rangle$ is an associated prime of $J(G)^{s}$.

Lemma 3.3. The following are equivalent:

(i) $P=\left\langle x_{i_{1}}, \ldots, x_{i_{r}}\right\rangle \in \operatorname{Ass}\left(J(G)^{s}\right)$ with $J(G) \subseteq k\left[x_{1}, \ldots, x_{n}\right]$

(ii) $P=\left\langle x_{i_{1}}, \ldots, x_{i_{r}}\right\rangle \in \operatorname{Ass}\left(J\left(G_{P}\right)^{s}\right)$ with $J\left(G_{P}\right) \subseteq k\left[x_{i_{1}}, \ldots, x_{i_{r}}\right]$.

Proof. The details are worked out in [25] using the properties of localization.

We take a detour to introduce some more graph theory: 
Definition 3.4. A graph $G$ is critically $s$-chromatic if $\chi(G)=s$, and for every $x \in V(G)$, $\chi(G \backslash\{x\})<s$.

Example 3.5. Let $G=C_{n}$ be the $n$-cycle with $n$ odd. Then $G$ is a critically 3 -chromatic graph since $\chi(G)=3$, but if we remove any vertex $x, \chi(G \backslash\{x\})=2$.

Example 3.6. Let $G=K_{n}$ be the clique of size $n$. Then $G$ is a critically $n$-chromatic graph since $\chi(G)=n$, but if we remove any vertex $x, G \backslash\{x\}=K_{n-1}$, and thus $\chi(G \backslash$ $\{x\})=n-1$.

Remark 3.7. You should be able to convince yourself that the only critically 1-chromatic graph is the graph of an isolated vertex, and the only critically 2-chromatic graph is $K_{2}$. The only critically 3 -chromatic graphs are precisely the graphs $G=C_{n}$ with $n$ odd. However, for $s \geq 4$, there is no known classification of critically $s$-chromatic graphs.

As the next theorem shows, some of the associated primes of $J(G)^{s}$ are actually detecting induced subgraphs that are critically $(s+1)$-chromatic.

Theorem 3.8. Let $G$ be a graph and suppose $P \subseteq V(G)$ is such that $G_{P}$ is critically $(s+1)$-chromatic. Then

(1) $P \notin \operatorname{Ass}\left(J(G)^{d}\right)$ for $1 \leq d<s$.

(2) $P \in \operatorname{Ass}\left(J(G)^{s}\right)$.

Proof. By Lemma 3.3, we can assume that $G=G_{P}$.

(1) Suppose that $P \in \operatorname{Ass}\left(J(G)^{d}\right)$ for some $d<s$. Thus, there exists some monomial $m \notin J(G)^{d}$ such that $J(G)^{d}:\langle m\rangle=P$.

We first note that $m \mid\left(x_{1} \cdots x_{n}\right)^{d-1}$. If not, then there is some $x_{i}$ such that $x_{i}^{d} \mid m$. Because $x_{i} m \in J(G)^{d}$, we can find $d$ vertex covers $W_{1}, \ldots, W_{d}$ such that $x_{i} m=x_{W_{1}} \cdots x_{W_{d}} M \in$ $J(G)^{d}$ for some monomial $M$. Since $x_{i}$ appears at least $d+1$ times on the left, it must appear the same number of times on the right. Because each $x_{W_{j}}$ is square-free, this means that $x_{i} \mid M$. We then have $m=x_{W_{1}} \cdots x_{W_{d}}\left(M / x_{i}\right) \in J(G)^{d}$, contradicting the fact that $m \notin J(G)^{d}$.

Because $J(G)^{d}:\langle m\rangle=P$, we have $x_{1} m \in J(G)^{d}$. Note that $x_{W}=x_{2} x_{3} \cdots x_{n} \in J(G)$ since $W=\left\{x_{2}, \ldots, x_{n}\right\}$ is a vertex cover. So, $x_{1} m x_{W} \in J(G)^{d+1}$, and moreover, $x_{1} m x_{W}$ will divide $\left(x_{1} \cdots x_{n}\right)^{d}$. So, $\left(x_{1} \cdots x_{n}\right)^{d} \in J(G)^{d+1}$. By Theorem 1.21, this means that $\chi(G) \leq d+1<s+1$. But this contradicts the fact that $\chi(G)=(s+1)$.

We now prove (2). We are given

$$
\chi(G)=\min \left\{t \mid\left(x_{1} \cdots x_{n}\right)^{t-1} \in J(G)^{t}\right\}=s+1
$$

so $m=\left(x_{1} \cdots x_{n}\right)^{s-1} \notin J(G)^{s}$. In other words, we have, $J(G)^{s}:\langle m\rangle \subsetneq\langle 1\rangle$, and hence $J(G)^{s}:\langle m\rangle \subseteq\left\langle x_{1}, \ldots, x_{n}\right\rangle$. We will now show that $J(G)^{s}:\langle m\rangle \supseteq\left\langle x_{1}, \ldots, x_{n}\right\rangle$; the conclusion will then follow from this fact.

Since $G$ is critically $(s+1)$-chromatic, for each $x_{i} \in V(G), \chi\left(G \backslash\left\{x_{i}\right\}\right)=s$. Let

$$
V\left(G \backslash\left\{x_{i}\right\}\right)=C_{1} \cup \cdots \cup C_{s}
$$


be the $s$ colouring of $V\left(G \backslash\left\{x_{i}\right\}\right)$. Then

$$
V(G)=C_{1} \cup \cdots \cup C_{s} \cup\left\{x_{i}\right\}
$$

is an $(s+1)$-colouring of $G$.

For $j=1, \ldots, s$, set

$$
W_{j}=C_{1} \cup \cdots \cup \widehat{C_{j}} \cup \cdots \cup C_{s} \cup\left\{x_{i}\right\} .
$$

Each $W_{j}$ is a vertex cover, so $x_{W_{j}} \in J(G)$. Thus

$$
\prod_{j=1}^{s} x_{W_{j}} \in J(G)^{s} .
$$

But $\prod_{j=1}^{s} x_{W_{j}}=\left(x_{1} \cdots x_{n}\right)^{s-1} x_{i}$. Thus, $x_{i} \in J(G)^{s}:\langle m\rangle$. This is true for each $x_{i} \in V(G)$, whence $\left\langle x_{1}, \ldots, x_{n}\right\rangle \subseteq J(G)^{s}:\langle m\rangle \subseteq\left\langle x_{1}, \ldots, x_{n}\right\rangle$, as desired.

Remark 3.9. It is believed (see Section 3.3 for more details) that Theorem 3.8 (2) can be strengthened to $\left(2^{\prime}\right) P \in \operatorname{Ass}\left(J(G)^{d}\right)$ for all $d \geq s$.

Example 3.10. We consider the following graph

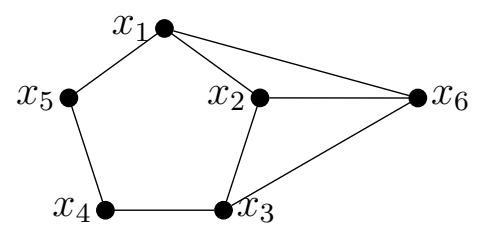

Note that the induced graph on $\left\{x_{1}, x_{2}, x_{6}\right\}$ is a $K_{3}$ (and $C_{3}$ ), a critically 3 -chromatic graph. So $P=\left\langle x_{1}, x_{2}, x_{6}\right\rangle$ is in $\operatorname{Ass}\left(J(G)^{2}\right)$, but not in $\operatorname{Ass}(J(G))$. Similarly, since the induced graph on $\left\{x_{1}, x_{2}, x_{3}, x_{4}, x_{5}\right\}$ is a $C_{5}$, we will have $\left\langle x_{1}, x_{2}, x_{3}, x_{4}, x_{5}\right\rangle \in \operatorname{Ass}\left(J(G)^{2}\right)$.

When $s=2$, we can find a converse of Theorem 3.8. In fact, we can give a complete characterization of the associated primes of $J(G)^{2}$; this result first appeared in [27].

Theorem 3.11. Let $G$ be a graph. A prime ideal $P=\left\langle x_{i_{1}}, \ldots, x_{i_{t}}\right\rangle \in \operatorname{Ass}\left(J(G)^{2}\right)$, if and only if:

(1) $P=\left\langle x_{i_{1}}, x_{i_{2}}\right\rangle$, and $\left\{x_{i_{1}}, x_{i_{2}}\right\} \in E(G)$, or

(2) $t$ is odd, and the induced graph on $\left\{x_{i_{1}}, x_{i_{2}}, \ldots, x_{i_{t}}\right\}$ is an induced cycle of $G$.

Example 3.12. By Theorem 3.11, we can write out all the elements of $\operatorname{Ass}\left(J(G)^{2}\right)$ for the graph $G$ of Example 3.10 .

$$
\begin{aligned}
\operatorname{Ass}\left(J(G)^{2}\right)= & \left\{\left\langle x_{1}, x_{2}\right\rangle,\left\langle x_{2}, x_{3}\right\rangle, \cdots,\left\langle x_{3}, x_{6}\right\rangle,\left\langle x_{1}, x_{2}, x_{6}\right\rangle,\left\langle x_{2}, x_{3}, x_{6}\right\rangle,\right. \\
& \left.\left\langle x_{1}, x_{2}, x_{3}, x_{4}, x_{5}\right\rangle,\left\langle x_{1}, x_{6}, x_{3}, x_{4}, x_{5}\right\rangle\right\}
\end{aligned}
$$

Remark 3.13. By Theorem 3.11, the associated primes of $J(G)^{2}$ are related to the odd induced cycles in the graph. This gives a method to identify all the odd induced cycles in a graph; in fact, this is the procedure used in the Macaulay 2 EdgeIdeals package. Since odd induced graphs play an important role in the classification of perfect graphs (see [7]), 
we can exploit the associated primes of $J(G)^{2}$ to determine if a graph is perfect. Again, see [27] for all the details.

Unfortunately, the converse of Theorem 3.8 is false in general; that is, if $P \in \operatorname{Ass}\left(J(G)^{s}\right)$, but $P \notin \operatorname{Ass}\left(J(G)^{d}\right)$ with $1 \leq d<s$, then the graph $G_{P}$ is not necessarily a critically $(s+1)$-chromatic graph.

Example 3.14. If we consider the graph of Example 3.10 , then $P=\left\langle x_{1}, x_{2}, x_{3}, x_{4}, x_{5}, x_{6}\right\rangle \in$ $\operatorname{Ass}\left(J(G)^{3}\right)$ but not in $\operatorname{Ass}(J(G))$ or $\operatorname{Ass}\left(J(G)^{2}\right)$. However, the graph $G=G_{P}$ is not critically 4-chromatic. In fact, $\chi(G)=3$.

What is happening here is that the colouring information in the associated primes is too "crude". We need to decompose the ideal $J(G)^{s}$ differently to extract the colouring information. The ideal $P=\left\langle x_{1}, x_{2}, x_{3}, x_{4}, x_{5}, x_{6}\right\rangle \in \operatorname{Ass}\left(J(G)^{3}\right)$ in the above example does come from a critically 4-chromatic graph, but it "lives" in a larger graph constructed from $G$. We expand upon this idea in the next section.

3.2. Powers of cover ideals: irreducible decomposition. Any monomial ideal of the form $\left\langle x_{i_{1}}^{a_{i_{1}}}, \ldots, x_{i_{t}}^{a_{i_{t}}}\right\rangle$ is an irreducible monomial ideal. A monomial ideal can then be decomposed into irreducible monomial ideals:

Theorem 3.15. Every monomial ideal I has a unique irredundant decomposition into irreducible ideals; i.e., we can write I uniquely as

$$
I=m_{1} \cap \cdots \cap m_{t}
$$

where each $m_{i}$ is an irreducible monomial ideal.

Proof. See [49, Theorem 5.27].

The next lemma is the basis of an algorithm to find this irreducible decomposition.

Lemma 3.16. Let I be a monomial ideal. If $m$ is a minimal generator of $I$ and $m=m_{1} m_{2}$ with $\operatorname{gcd}\left(m_{1}, m_{2}\right)=1$, then

$$
I=\left(I+\left\langle m_{1}\right\rangle\right) \cap\left(I+\left\langle m_{2}\right\rangle\right)
$$

Example 3.17. If $I=\left\langle x^{2}, x y, y^{2}\right\rangle$, we can decompose it as

$$
I=\left\langle x^{2}, x y, y^{2}, x\right\rangle \cap\left\langle x^{2}, x y, y^{2}, y\right\rangle=\left\langle x, y^{2}\right\rangle \cap\left\langle x^{2}, y\right\rangle .
$$

This example gives a hint of why an irreducible decomposition will be more useful. Note that if $J=\langle x, y\rangle$, then $I=J^{2}$. Then not only can we read off the associated primes, e.g., $\operatorname{Ass}\left(J^{2}\right)=\{\langle x, y\rangle\}$, but the irreducible decomposition is another way to express the original ideal.

We now take a detour to introduce some more graph theory: 
Definition 3.18. Given a graph $G=(V(G), E(G))$ and integer $s \geq 1$, the $s$-th expansion of $G$, denoted $G^{s}$, is the graph constructed from $G$ as follows: (a) replace each $x_{i} \in V(G)$ with a clique of size $s$ on the vertices $\left\{x_{i, 1}, \ldots, x_{i, s}\right\}$, and (b) two vertices $x_{i, a}$ and $x_{j, b}$ are adjacent in $G^{s}$ if and only if $x_{i}$ and $x_{j}$ were adjacent in $G$.

Example 3.19. We illustrate this example when $G=C_{4}$, and we construct $G^{2}$. Recall that $C_{4}$ is the graph:

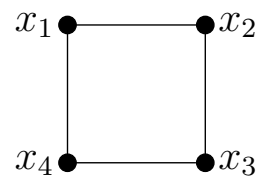

Then the second expansion of $G$ is the graph:

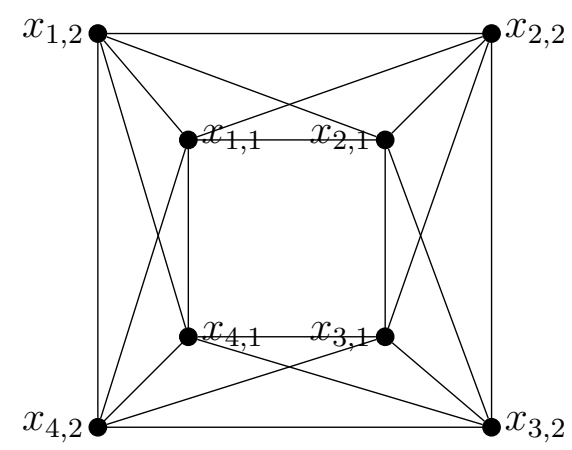

We now come to our main result:

Theorem 3.20. Let $G$ be a graph with cover ideal $J(G)$. Then $\left\langle x_{i_{1}}^{a_{i_{1}}}, \ldots, x_{i_{r}}^{a_{i_{r}}}\right\rangle$ appears in the irreducible decomposition of $J(G)^{s}$ if and only if the induced graph on

$$
\left\{x_{i_{1}, 1}, \ldots, x_{i_{1}, s-a_{i_{1}}+1}, \ldots, x_{i_{r}, 1}, \ldots, x_{i_{r}, s-a_{i_{r}}+1}\right\}
$$

in $G^{s}$ is a critically $(s+1)$-chromatic graph.

The proof is a mixture of a number of ingredients. It relies on generalized Alexander duality, polarization and depolarization of monomial ideals, and a result of Sturmfels and Sullivant [60]. We have only stated it for edge ideals of graphs, but it works also for edge ideals of hypergraphs, i.e., any square-free monomial ideal.

Example 3.21. Let us return to Example 3.10 and explain why $\left\langle x_{1}, x_{2}, x_{3}, x_{4}, x_{5}, x_{6}\right\rangle$ appears in $\operatorname{Ass}\left(J(G)^{3}\right)$. If we look at the irreducible decomposition of $J(G)^{3}$ (which we can compute using Macaulay 2), one of the irreducible monomial ideals that appears is the ideal

$$
\left\langle x_{1}^{3}, x_{2}^{3}, x_{3}^{3}, x_{4}^{3}, x_{5}^{2}, x_{6}^{3}\right\rangle .
$$

So, we need to look at the induced graph on

$$
\left\{x_{1,1}, x_{2,1}, x_{3,1}, x_{4,1}, x_{5,1}, x_{5,2}, x_{6,1}\right\}
$$


in $G^{3}$. This graph looks like:

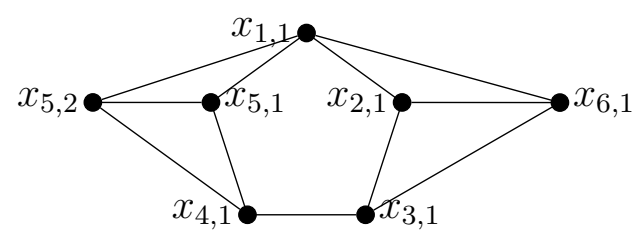

You can now convince yourself that this graph is critically 4-chromatic.

3.3. Persistence of primes and a conjecture. We end with a conjecture about the persistence of primes.

Definition 3.22. An ideal $I$ in a Noetherian $\operatorname{ring} R$ has the persistence property if

$$
\operatorname{Ass}\left(I^{s}\right) \subseteq \operatorname{Ass}\left(I^{s+1}\right) \text { for all } s \geq 1
$$

Not every ideal has the persistence property, and in fact, proving that an ideal has this property can be quite difficult. With respect to edge and cover ideals, we have the following results:

Theorem 3.23. (a) (see [48]) For any graph $G$, the edge ideal $I(G)$ has the persistence property.

(b) (see [25]) If $G$ is a chordal graph ${ }^{6}$, then $J(G)$ has the persistence property.

What is interesting about the proof of $(a)$ found in [48] is that one needs a classical result from graph theory due to Berge on matchings in a graph to prove the persistence property. The paper [48] is a good example of using results from graph theory to prove an interesting algebraic result.

Computer experiments have suggested that Theorem $3.23(b)$ will hold for any graph $G$, i.e., the cover ideal $J(G)$ for any $G$ also has the persistence property. Unlike the case of edge ideals, we need a graph theory result that appears unknown. We state the "missing" graph theory result.

Definition 3.24. Let $W \subseteq V(G)$. The expansion of $G$ at $W$, denoted $G[W]$ is the the graph obtained by replacing $x_{i} \in W$ with the edge $\left\{x_{i, 1}, x_{i, 2}\right\}$ and joining to these two vertices all the vertices to which $x_{i}$ was joined.

The following conjecture is found in [26]:

Conjecture 3.25. Suppose that $G$ is a critically s-chromatic graph. Then there exists a $W \subseteq V(G)$ such that $G[W]$ is a critically $(s+1)$-chromatic graph.

Example 3.26. The conjecture is true for all odd cycles (i.e., the critically 3-chromatic graphs; see [26] for a proof) and for graphs whose fractional chromatic number $\chi_{f}(G)$ (see Section 1) is "close" to $\chi(G)$, i.e., $\chi(G)-1<\chi_{f}(G) \leq \chi(G)$. We illustrate the conjecture

\footnotetext{
${ }^{6}$ The result holds for a larger class of graphs called perfect graphs.
} 
with the graph $G=C_{5}$. If we expand this graph at the vertices $W=\left\{x_{2}, x_{5}\right\}$, we get the graph

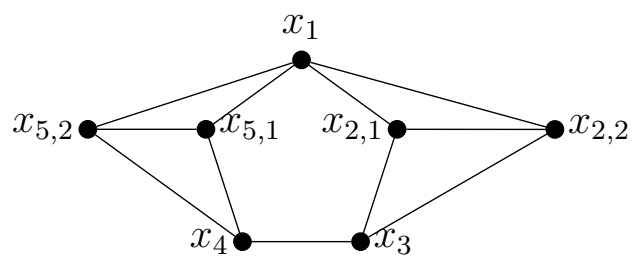

This graph is critically 4-chromatic.

Francisco, Hà, and myself then proved that if this graph theory conjecture is true, then one could prove that every cover ideal has the persistence property.

Theorem 3.27. If Conjecture 3.25 is true, then every cover ideal $J(G)$ has the persistence property.

Since the conjecture is true for $s=2$ and $s=3$, we know that for all graphs $G$, we have the following containments:

$$
\operatorname{Ass}(J(G)) \subseteq \operatorname{Ass}\left(J(G)^{2}\right) \subseteq \operatorname{Ass}\left(J(G)^{3}\right) .
$$

We end with an open ended question. For any hypergraph, one can formulate a hypergraph version of Conjecture 3.25 and Theorem 3.20. Since the cover ideals of hypergraphs are in one-to-one correspondence with all square-free monomial ideals, do we really have:

Question 3.28. Is it true that all square-free monomial ideals have the persistence property?

\section{The Macaulay 2 PACKAGE EdgeIdeals}

Computer algebra systems, like Macaulay 2 [31, Singular [14, and CoCoA 8, have become essential tools for many mathematicians in commutative algebra and algebraic geometry. These systems provide a "laboratory" in which we can experiment and play with new ideas. From these experiments, a researcher can formulate new conjectures, and hopefully, new theorems. Computer algebra systems are especially good at dealing with monomial ideals. As a consequence, the study of edge and cover ideals is well suited to experiments using computer algebra systems.

The purpose of this section is to familiarize the user with the package EdgeIdeals that was written by C. Francisco, A. Hoefel, and myself [29]. This package, written for Macaulay 2, provides a suite of functions to experiment with edge and cover ideals. Many of the results discussed in the notes have been implemented into this package. Hopefully, the tools introduced in this tutorial will be the basis of your own research results!

As a final note, although I primarily discuss the EdgeIdeal package, I would recommend that your also become familiar with the packages SimplicialComplexes, written by S. Popescu, G.G. Smith, and M. Stillman (see [42]), and SimplicialDecomposability by 
D.W. Cook II (see [10]). The first package contains a number of useful functions related to simplicial complexes. In fact, the EdgeIdeals package requires a number of functions from this package. The SimplicialDecomposability package of D.W. Cook II is useful if you wish to study the properties of the simplicial complex associated to the edge or cover ideal of a graph.

4.1. Getting Started. Obviously, the first thing you need to do is install the latest version $]^{7}$ of Macaulay 2 on your computer. The download page is here:

$$
\text { http : //www.math.uiuc.edu/Macaulay2/Downloads/ }
$$

Pick the appropriate operating system, and then follow the instructions. This may take some time and patience.

I am going to assume that you have installed Macaulay 2 and now have it working. To familiarize yourself with the basic syntax and some simple examples, a good place to start is this web page:

$$
\text { http : //www.math.uiuc.edu/Macaulay2/GettingStarted/ }
$$

If you have never used Macaulay 2, take a couple of minutes to try a couple of the sample sessions.

4.2. The EdgeIdeals Package. Now that you have Macaulay 2 installed, we want to load the EdgeIdeals package. If you are using a current version of Macaulay 2 (i.e., a version $\geq 1.2$ ), then this package should already be included with your installation of Macaulay 2, and it simply has to be installed.

Remark 4.1. If you have an older version, or if your version does not include this package, you should first download the source code from this link:

$$
\text { http ://j - sag.org/Volume1/EdgeIdeals.m2 }
$$

Save the code in a file named EdgeIdeals.m2, and save the file into your working directory. You can now return to the directions below. Note that when you run the command installPackage 'EdgeIdeals', Macaulay 2 will install the package where it can always find it in the future.

Open Macaulay 2 and input the following command

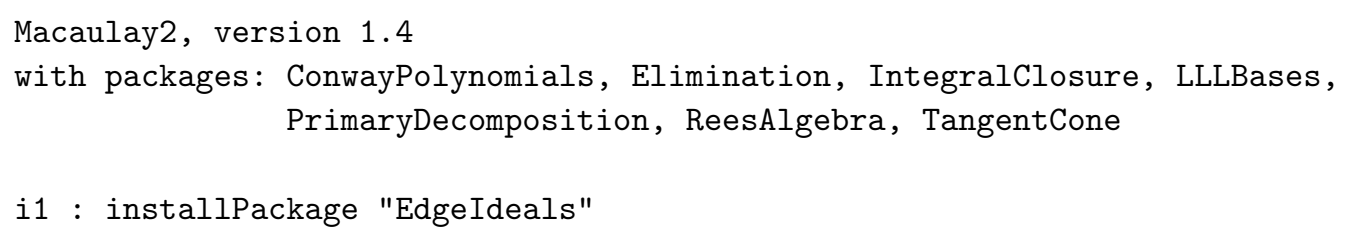

${ }^{7}$ At the time of writing this tutorial, the current version was 1.4 
You will only need to enter this command the first time you use the package. In the background, this command is making all the help pages. Once you have installed the package, you do not need to use the command again, but instead, use the instructions below. If you wish, you can start a new session by typing restart.

When we first start Macaulay 2, we start with following screen:

Macaulay2, version 1.4

with packages: ConwayPolynomials, Elimination, IntegralClosure, LLLBases, PrimaryDecomposition, ReesAlgebra, TangentCone

i1:

At the prompt, type the following command to load the package EdgeIdeals:

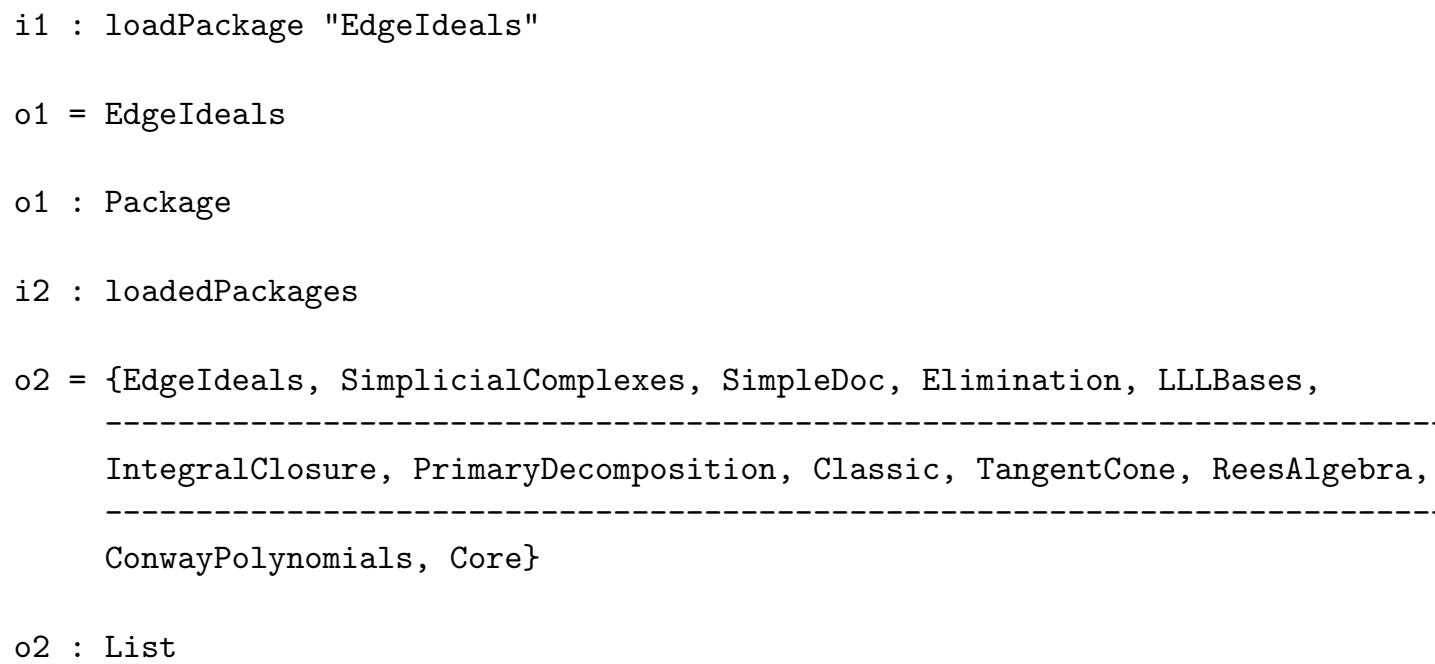

The second command returns all the packages currently loaded in Macaulay 2. Note that not only is the EdgeIdeals package loaded, but so is the SimplicialComplexes package. Many of the functions in EdgeIdeals run "on top" of SimplicialComplexes.

We are now ready to try out EdgeIdeals. To get going, we spend a little time discussing how to input a finite simple graph. As a concrete example, suppose that we want to study the graph

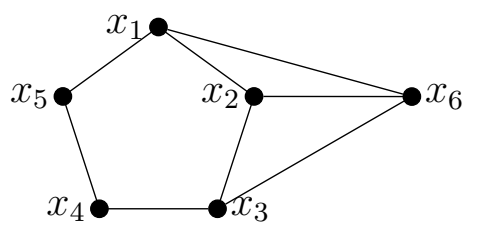

We enter this information in such a way that Macaulay 2 recognizes it as a graph. There are a couple of ways to do this. The first way is to input a polynomial ring to denote the vertices, and then represent the edges as a list. For example

i3 $: R=Q Q\left[x_{-} 1 \ldots x_{-} 6\right]$

$03=R$ 


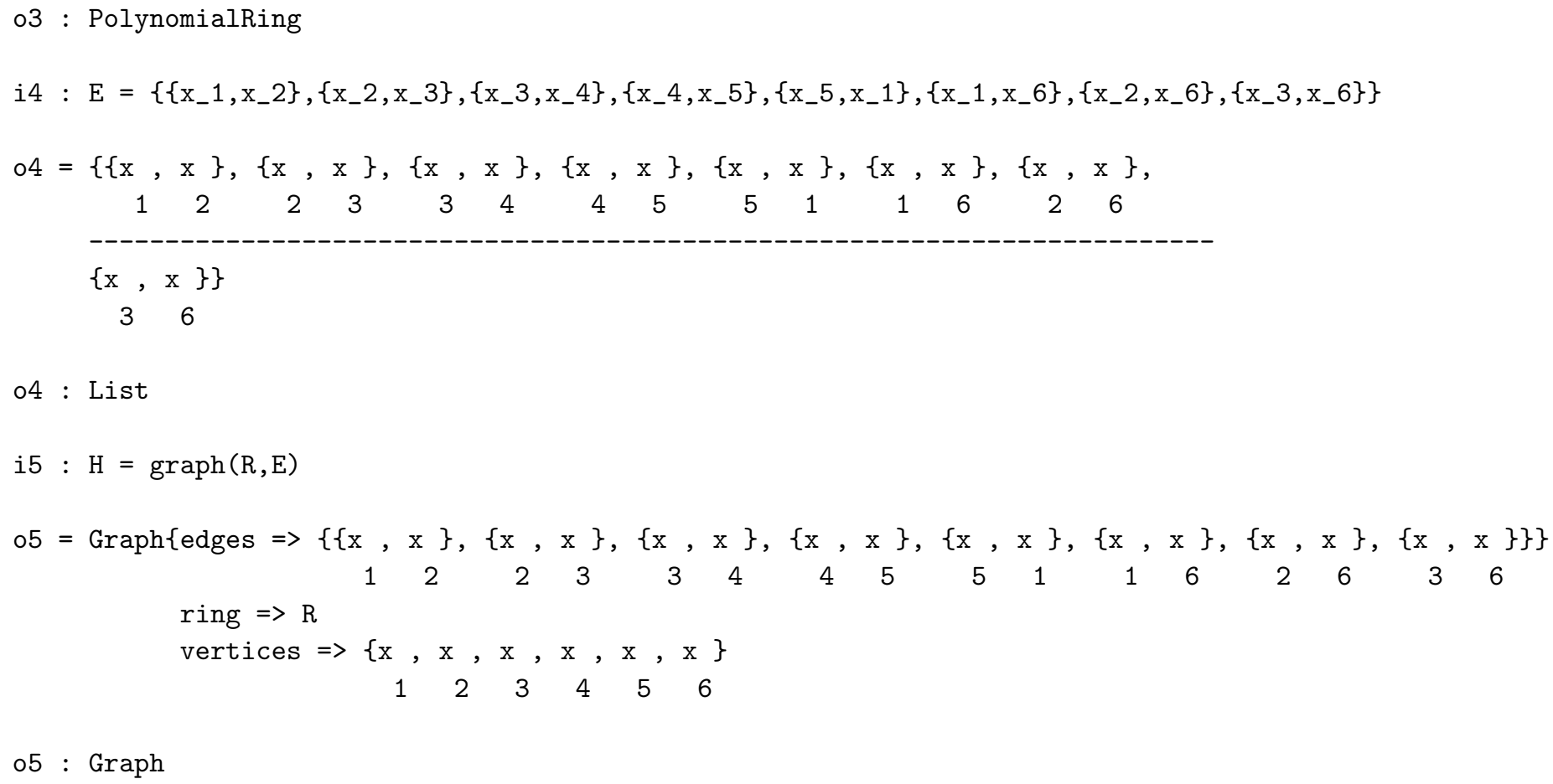

Alternatively, the edges can be represented as the generators of a square-free quadratic monomial ideal. If no ring is passed to the command graph, it takes the variables of the current ring as the vertices of the graph. As an example, here is an alternative way to input the above graph into Macaulay 2:

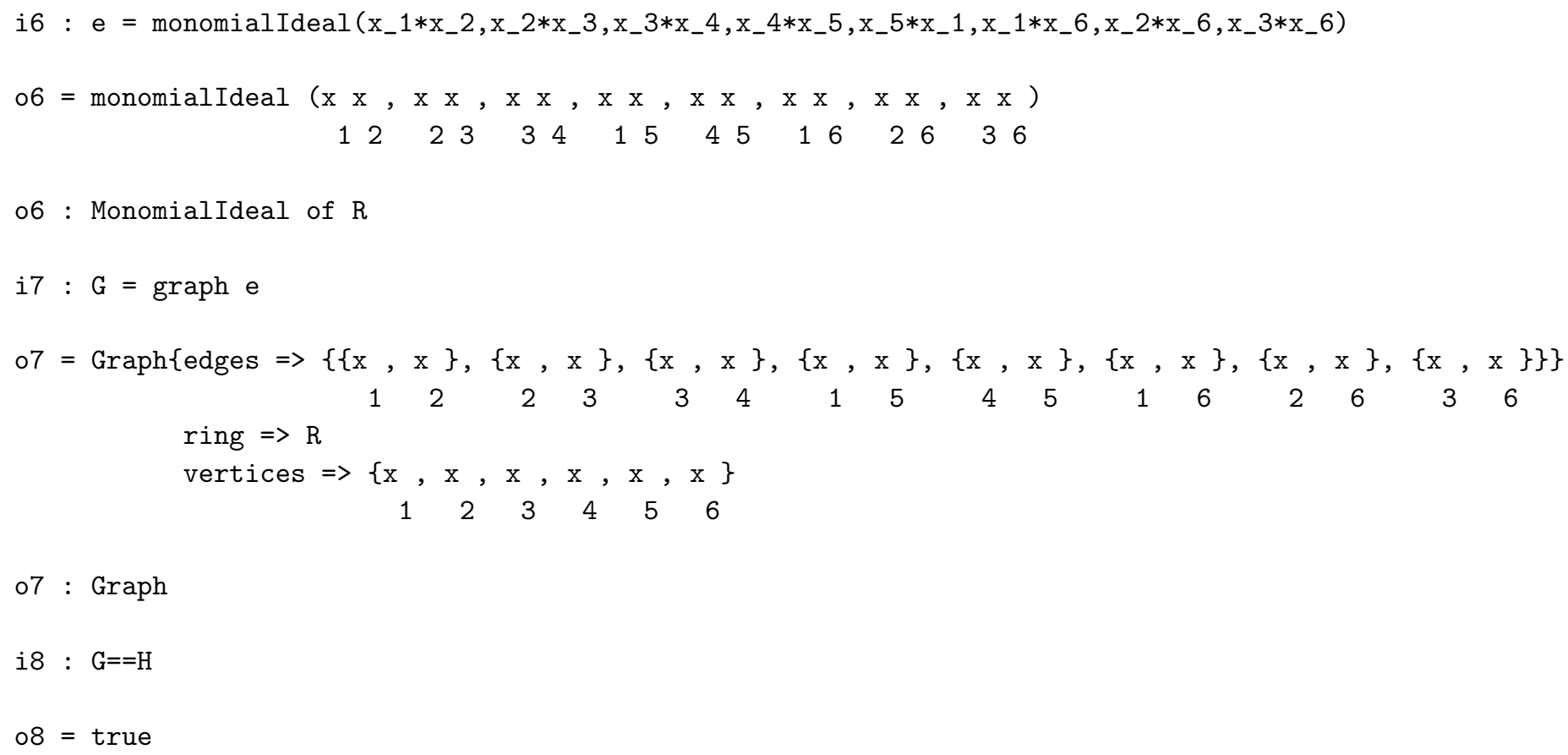


Now that we have an object called a Graph, we can ask about its edge and cover ideals. Both of these ideals can be easily obtained using the following commands:

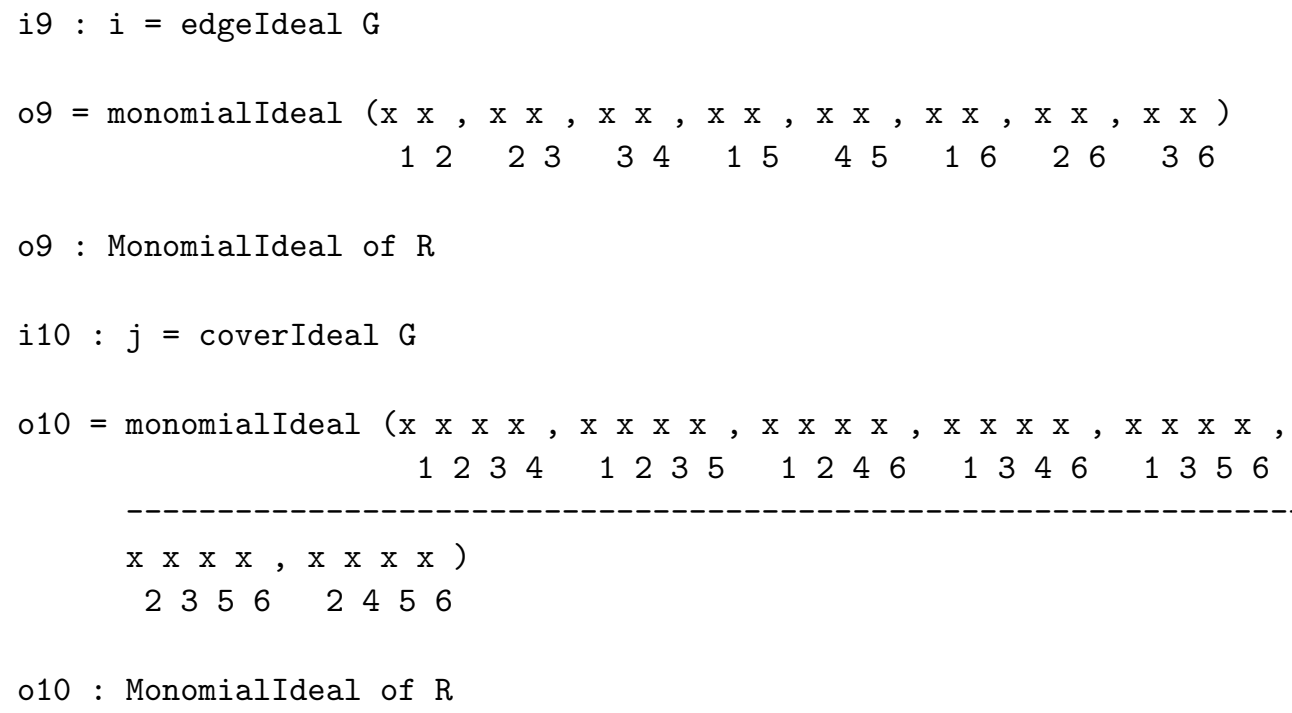

The generators of $J(G)$ are the minimal vertex covers of $G$; convince yourself that the generators given in the above example are indeed the minimal vertex covers of the graph.

Recall that we showed that the Alexander dual of the edge ideal $I(G)$ equals the cover ideal of $J(G)$. We can verify this for this ideal using a command from the SimplicialComplexes package (which is also loaded):

i11 : dual $i==j$

$011=$ true

Once you have inputted your graph, you can now compute some of its graph theoretic invariants. For example, the chromatic number of the graph is computed as

i12 : chromaticNumber G

$012=3$

To compute this number, we use the fact that

$$
\chi(G)=\min \left\{d \mid\left(x_{1} \cdots x_{n}\right)^{d-1} \in J(G)^{d}\right\}
$$

as proved in Section 1. Similarly, Fröberg's Theorem gives us an algebraic characterization of chordal graphs. We can therefore check if $G$ is chordal:

i13 : isChordal G

$013=$ false

To facilitate experimentation, we have built a number of functions to create commonly occurring graphs, like cycles and cliques. Here are some examples:

i14: $\mathrm{C6}=$ cycle $\mathrm{R}$ 


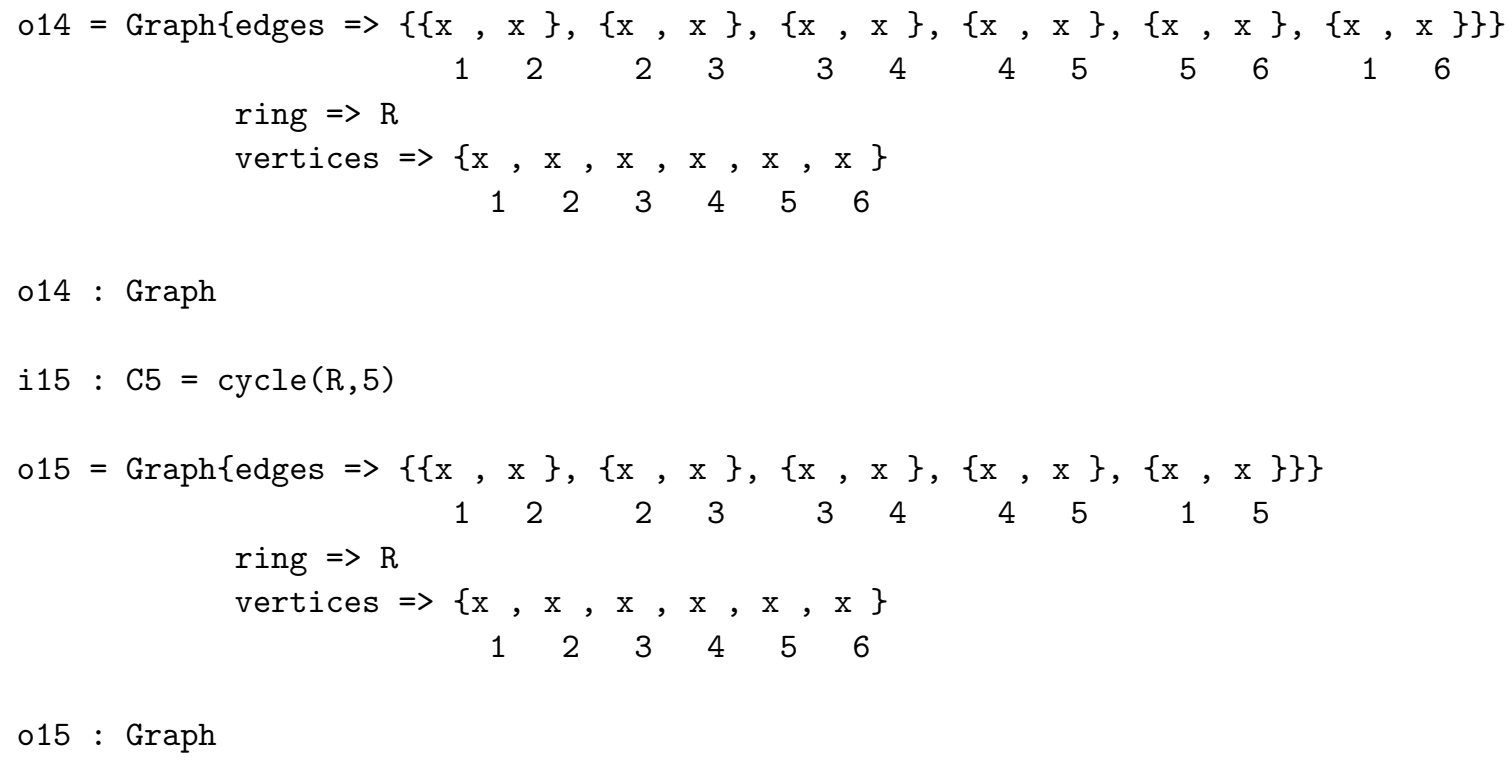

The command cycle will return a cycle of length equal to the number of variables in the ring $\mathrm{R}$ as a default. If a number $n$ is given, it will make a cycle of that length using the first $n$ variables. Cliques of size $n$ are defined similarly:

i16: $\mathrm{K} 4=\operatorname{completeGraph}(\mathrm{R}, 4)$

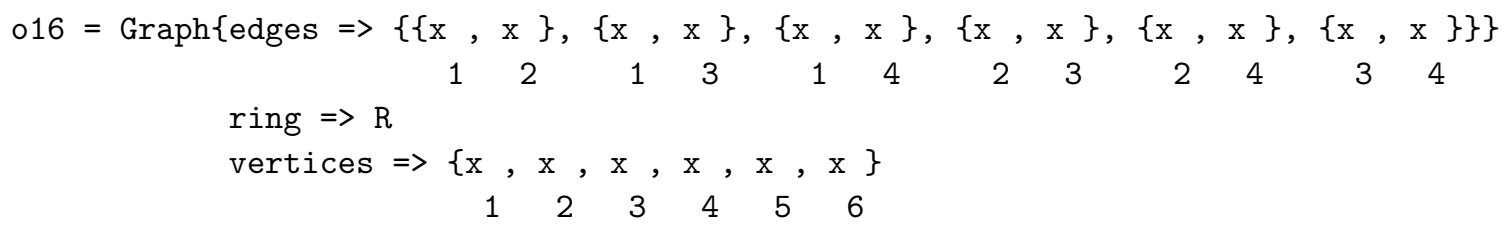

The command antiCycle is similar in that it returns the graph of the complement of a cycle.

Also built into the EdgeIdeals package is a number of commands to construct subgraphs. For example, suppose that we wish to look at the induced subgraph of $G$ on the vertices $P=\left\{x_{1}, x_{2}, x_{6}, x_{5}\right\}$. This can be done as follows:

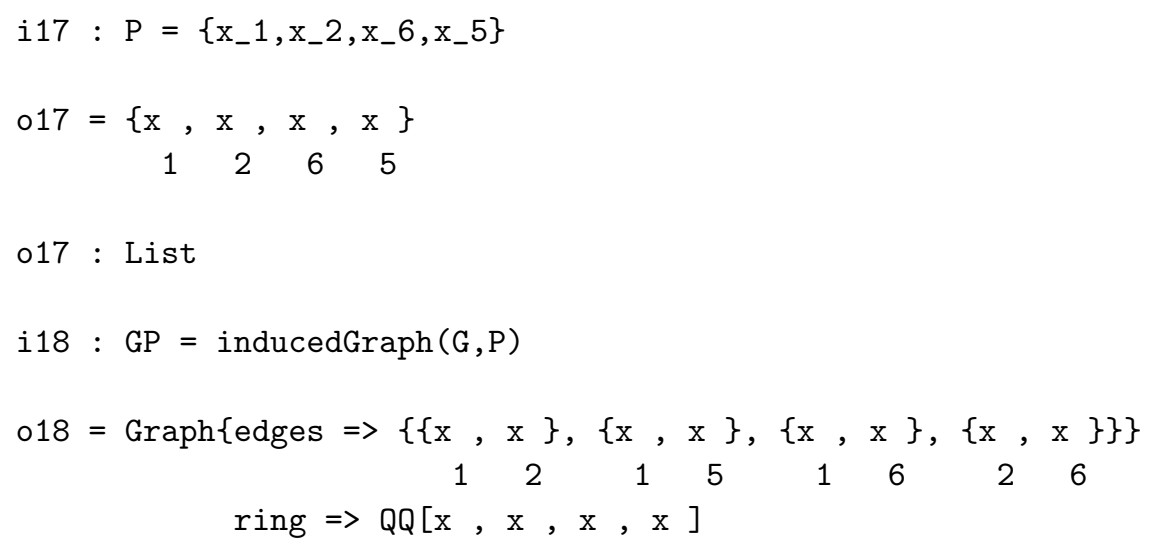


018 : Graph

Another similar command that may prove helpful is deleteEdges which removes a collection of edges from a graph.

To facilitate research, the EdgeIdeals package includes a function called randomGraph. This function allows you to generate a random graph on defined number of vertices and edges, and is useful when creating conjectures. Here is an example of the this function in action:

i19 : randomGraph $(\mathrm{R}, 8)$

$019=\operatorname{Graph}\{$ edges $\Rightarrow\{\{\mathrm{x}, \mathrm{x}\},\{\mathrm{x}, \mathrm{x}\},\{\mathrm{x}, \mathrm{x}\},\{\mathrm{x}, \mathrm{x}\},\{\mathrm{x}, \mathrm{x}\},\{\mathrm{x}, \mathrm{x}\},\{\mathrm{x}, \mathrm{x}\},\{\mathrm{x}, \mathrm{x}\}\}\}$

ring $\Rightarrow \mathrm{R}$

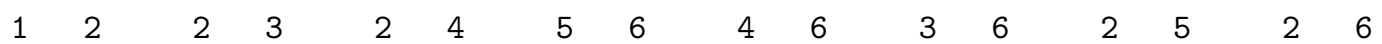

vertices $=>\{x, x, x, x, x, x\}$

$\begin{array}{llllll}1 & 2 & 3 & 4 & 5 & 6\end{array}$

019 : Graph

In this case, we are asking for a random graph on 6 vertices (the number of variables in the polynomial ring $R$ ) with 8 edges. This function can be used to test a large number of examples quickly.

As a final note, the documentation of the EdgeIdeals package can be found here:

http://www.math.uiuc.edu/Macaulay2/doc/Macaulay2-1.4/share/doc/Macaulay2/EdgeIdeals/html/index.html

All the commands given in the package are listed on this page. Detailed documentation and examples can be found by clicking on the appropriate links.

\section{TUtORIAls}

I have included two tutorials to give you a chance to play around and experiment with edge and cover ideals using Macaulay 2. These tutorials were first given to the participants of MONICA. When required, the tutorials provide needed definitions, results, and references. Some of the initial problems ask you to prove some simple results in order to give you a feeling for the material, while other problems ask you to program some simple procedures using Macaulay 2 in order to help you develop your Macaulay 2 skills. The last batch of questions for each tutorial is a series of open questions. These questions are denoted by an asterisk. (If you come up with any ideas, I would love to hear them!)

5.1. Tutorial 1: Splitting Monomial Ideals. In this tutorial, we explore some of the properties of splitting monomial ideals as discussed in Section 2. 
Exercise 5.1.1. Suppose $I=J+K$ is a Betti splitting. Prove that

$$
\operatorname{reg}(I)=\max \{\operatorname{reg}(J), \operatorname{reg}(K), \operatorname{reg}(J \cap K)-1\} .
$$

Here, $\operatorname{reg}(-)$ denotes the regularity of the given ideal.

Remark. This result can be quite useful when doing induction. For example, this fact was used to give a new proof for the regularity of the edge ideal of a tree [34].

Exercise 5.1.2. Write a Macaulay 2 program that takes as input two monomial ideals $J$ and $K$, and will return true or false depending upon whether $J+K$ is a Betti splitting.

Hint. The command betti res I will return the Betti diagram of the ideal $I$. Read through the betti documentation in order to extract out the information you are looking for. If you are interested in a particular graded Betti number, you may wish to first define the function:

beta $=(i, j, I) \rightarrow($ betti res $I) \#(i,\{j\}, j)$

Exercise 5.1.3. (Importance of $\operatorname{char}(k))$ Consider the following ideal in $R=k\left[x_{1}, \ldots, x_{6}\right]$ :

$$
I=\left(x_{1} x_{2} x_{4}, x_{1} x_{2} x_{6}, x_{1} x_{3} x_{5}, x_{1} x_{3} x_{4}, x_{1} x_{5} x_{6}, x_{2} x_{4} x_{5}, x_{2} x_{3} x_{6}, x_{2} x_{3} x_{5}, x_{3} x_{4} x_{6}, x_{4} x_{5} x_{6}\right) .
$$

Fix a variable $x_{i}$, and form an $x_{i}$-partition of $I$, i.e., let $J$ be the ideal generated by all the generators of $I$ divisible by $x_{i}$, and let $K$ be the ideal generated by the remaining generators. Use Macaulay 2 to show $I=J+K$ is a Betti splitting in $\operatorname{char}(k)=2$, but not a Betti splitting if $\operatorname{char}(k) \neq 2$.

Hint. One way to input a ring of characteristic two is

i1 : $S=Z Z /(2)[a, b, c]$

Definition 5.1. Let $I(G)$ be the edge ideal of a graph. For any edge $e=\left\{x_{i}, x_{j}\right\}$, we have the partition

$$
I(G)=\left\langle x_{i} x_{j}\right\rangle+I(G \backslash e)
$$

where $G \backslash e$ is the graph $G$ with the edge $e$ removed. We call $e$ a splitting edge if this partition is a Betti splitting.

Exercise 5.1.4. Consider the graph

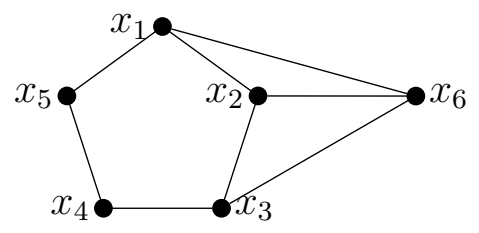

Determine which edges of this graph are splitting edges.

Exercise 5.1.5. Write a program in Macaulay 2 that inputs a graph, and returns all the edges in the graph that are splitting edges.

Exercise 5.1.6. Let $G=C_{n}$ be a cycle of length $n \geq 4$. Prove that $G$ has no splitting edge. 
Exercise 5.1.7. Let $G=K_{n}$ be the clique of size $n \geq 3$. Prove that every edge of $G$ is a splitting edge.

Exercise 5.1.8. Find a graph $G$ that is not a cycle, but no edge is a splitting edge, or prove that this is not possible. Then, find a graph $G$ that is not a clique, but every edge is a splitting edge, or prove that this is not possible.

Exercise 5.1.9. A vertex $v$ is called a leaf if $\operatorname{deg} v=1$. Suppose that $v$ is a leaf, and $e=\{v, u\}$ is the only edge that contains $v$. Prove that $e$ is a splitting edge.

Exercise 5.1.10. Let $N(x)=\{y \mid\{x, y\} \in E(G)\}$ be the neighbours of $x$. Make a conjecture about $\{x, y\}$ being a splitting edge in terms of $N(x) \cup N(y)$. Compare your answer to [34].

$\star$ Exercise 5.1.11. Is the number of splitting edges related to any invariants of $G$ or $I(G)$ ?

$\star$ Exercise 5.1.12. Find other ways to split $I(G)$.

$\star$ Exercise 5.1.13. Are there any nice ways to construct Betti splittings of the cover ideal $J(G)$ ? (I am only aware of how to split $J(G)$ in the case that $R / J(G)$ is Cohen-Macaulay and $G$ is bipartite [28].)

$\star$ Exercise 5.1.14. Are there Betti splittings of the ideals $I(G)^{s}$ and $J(G)^{s}$, for some integer $s$ ?

5.2. Tutorial 2: Regularity. In this tutorial, we look at the regularity of edge and cover ideals.

Exercise 5.2.1. A tree is a graph without any induced cycles. If $T$ is a tree, what is the regularity of $I\left(T^{c}\right)$, where $T^{c}$ is the complement of $T$ ?

Exercise 5.2.2. Describe all trees $T$ with the property that $\operatorname{reg}(I(T))=2$.

Exercise 5.2.3. Create any graph $G$ where the smallest induced cycle of $G^{c}$ has length 4. Use Macaulay 2 to compute the resolution. Now repeat for a graph $G$ whose smallest induced cycle in $G^{c}$ has length $5,6,7, \ldots$ until you observe your pattern. Compare your answer to Eisenbud, et al. [17].

Exercise 5.2.4. If you would like to see the code of a Macaulay 2 function, you can use

$$
\text { code methods use 〈function name〉 }
$$

Look at the code for smallestCycleSize. Try to figure out how Macaulay 2 finds the smallest induced cycle in a graph.

Exercise 5.2.5. Write a Macaulay 2 function that checks if a graph has an induced 4 cycle.

Hint. Use the fact that

$$
\beta_{1,4}(I(G))=c_{4}\left(G^{c}\right)
$$

where $c_{4}(H)$ denotes the number of induced four cycles in the graph $H$ (see [64]). 
Exercise 5.2.6. Write a Macaulay 2 function that tests whether an ideal has a linear resolution.

Exercise 5.2.7. Nevo and Peeva [53] have made the following conjecture:

Conjecture 5.2. For all graphs $G$, if $G^{c}$ has no induced four cycles, then there exists an integer s such that $I(G)^{\text {s }}$ has a linear resolution.

Using the command randomGraph, find 10 graphs where the conjecture is true, and for each graph, find the smallest integer $s$ where $I(G)^{s}$ has a linear resolution.

Exercise 5.2.8. The path of length $n$, denoted $P_{n}$ is the graph with vertex set $\left\{x_{1}, x_{2}, \ldots, x_{n}\right\}$ and edge set

$$
\left\{\left\{x_{1}, x_{2}\right\},\left\{x_{2}, x_{3}\right\}, \ldots,\left\{x_{n-1}, x_{n}\right\}\right\} .
$$

Compute the regularity of $I\left(P_{n}\right)$ for some $n$ until you find a pattern. Compare your result to Jacques [47.

$\star$ Exercise 5.2.9. Let $T$ be a tree. Find a formula for $\operatorname{reg}\left(I(T)^{s}\right)$ as $s$ varies.

Hint. You may wish to start with the case that $T=P_{n}$ first.

$\star$ Exercise 5.2.10. Find a formula for $\operatorname{reg}(J(G))$ and $\operatorname{reg}(I(G))$ for any graph.

Hint. This problem is probably too open ended. I am not aware of many results on the regularity of $J(G)$. For edge ideals, more is known (do a Google search on "regularity edge ideals"). For bipartite graphs, we almost have a complete story. See [62] for more.

\section{REFERENCES}

[1] A. Alilooee, S. Faridi, Betti numbers of path ideals of cycles and line. Preprint (2011) arXiv: $1110.6653 \mathrm{v} 113$

[2] J. Biermann, Cellular structure on the minimal resolution of the edge ideal of the complement of the $n$-cycle. Preprint (2011). 23

[3] R. Bouchat, H.T. Hà, A. O'Keefe, Path ideals of rooted trees and their graded Betti numbers. J. Combin. Theory Ser. A 118 (2011), 2411-2425. 13

[4] W. Bruns, J. Herzog, Cohen-Macaulay rings. Cambridge Studies in Advanced Mathematics, 39. Cambridge University Press, Cambridge, 1993. 3, 10

[5] J. Chen, S. Morey, A. Sung, The stable set of associated primes of the ideal of a graph. Rocky Mountain J. Math. 32 (2002), no. 1, 71-89. 24

[6] R.-X. Chen, Minimal free resolutions of linear edge ideals. J. Algebra, 324 (2010), no. 12, 3591-3613. 23

[7] M. Chudnovsky, N. Robertson, P. Seymour, R. Thomas, The strong perfect graph theorem. Ann. of Math. (2) 164 (2006), 5 1-229. 26

[8] CoCoATeam, CoCoA: a system for doing Computations in Commutative Algebra. Available at http://cocoa.dima.unige.it 30

[9] A. Conca, E. De Negri, M-Sequences, graph ideals and ladder ideals of linear type. J. Algebra 211 (1999), no. 2, 599-624. 13

[10] D.W. Cook II, Simplicial Decomposability. J. Software Algebra Geom. 2 (2010) 20-23. 31

[11] A. Corso and U. Nagel, Specializations of Ferrers ideals. J. Algebraic Comb. 28 (2008), 425-437. 23 
[12] A. Corso, U. Nagel, Monomial and toric ideals associated to Ferrers graphs. Trans. Amer. Math. Soc. 361 (2009), no. 3, 1371-1395. 23

[13] K. Dalili, M. Kummini, Dependence of Betti Numbers on Characteristic. Preprint (2010) arXiv: $1009.4243 \mathrm{v} 114$

[14] W. Decker, G.-M. Greuel, G. Pfister, H. Schönemann, Singular 3-1-3 - A computer algebra system for polynomial computations. http://www.singular.uni-kl.de (2011). 30

[15] A. Dochtermann, A. Engström, Algebraic properties of edge ideals via combinatorial topology. Electron. J. Combin. 16 (2009), no. 2, Special volume in honor of Anders Bjorner, Research Paper 2, 24 pp. 16

[16] A. Dochtermann, A. Engström, Cellular resolutions of cointerval ideals. Math. Z. 270 (2012), 145163. 23

[17] D. Eisenbud, M. Green, K. Hulek, S. Popescu, Restricting linear syzygies: algebra and geometry. Compos. Math. 141 (2005), no. 6, 1460-1478. 16, 21, 38

[18] S. Eliahou, M. Kervaire, Minimal resolutions of some monomial ideals. J. Algebra 129 (1990), no. 1, 1-25. 2, 18, 22

[19] E. Emtander, Betti numbers of hypergraphs. Comm. Algebra 37 (2009), no. 5, 1545-1571. 13

[20] E. Emtander, A class of hypergraphs that generalizes chordal graphs. Preprint (2008) arXiv:0803.2150v2 21

[21] S. Faridi, The facet ideal of a simplicial complex. Manuscripta Math. 109 (2002), 159-174. 13

[22] G. Fatabbi, On the resolution of ideals of fat points. J. Algebra 242 (2001), no. 1, 92-108. 22

[23] O. Fernández-Ramos, P. Gimenez, First nonlinear syzygies of ideals associated to graphs. Comm. Algebra 37 (2009), no. 6, 1921-1933. 21

[24] C.A. Francisco, Resolutions of small sets of fat points. J. Pure Appl. Algebra 203 (2005), no. 1-3, 220-236. 22

[25] C.A. Francisco, H.T. Hà, and A. Van Tuyl, Colorings of hypergraphs, perfect graphs, and associated primes of powers of monomial ideals. J. Algebra 331 (2011), 224-242. 8, 10, 13, 24, 29

[26] C.A. Francisco, H.T. Hà, and A. Van Tuyl, A conjecture on critical graphs and connections to the persistence of associated primes. Discrete Math. 310 (2010), no. 15-16, 2176-2182. 29

[27] C.A. Francisco, H.T. Hà, and A. Van Tuyl, Associated primes of monomial ideals and odd holes in graphs. J. Algebraic Combin. 32 (2010), no. 2, 287-301. 26, 27

[28] C.A. Francisco, H.T. Hà, A. Van Tuyl, Splittings of monomial ideals. Proc. Amer. Math. Soc. 137 (2009), no. 10, 3271-3282. 18, 22, 38

[29] C.A. Francisco, A. Hoefel, A. Van Tuyl, EdgeIdeals: a package for (hyper)graphs. J. Software Algebra Geom. 1 (2009) 1-4.2, 9,30

[30] R. Fröberg, On Stanley-Reisner rings. Topics in algebra, Part 2 (Warsaw, 1988), 57-70, Banach Center Publ., 26, Part 2, PWN, Warsaw, 1990. 2, 15, 16

[31] D. R. Grayson and M. E. Stillman, Macaulay 2, a software system for research in algebraic geometry. http://www.math.uiuc.edu/Macaulay2/9, 30

[32] H.T. Hà, A. Van Tuyl, Monomial ideals, edge ideals of hypergraphs, and their graded Betti numbers. J. Algebraic Combin. 27 (2008) 215-245. $13,21,22,23$

[33] H.T. Hà, A. Van Tuyl, Resolutions of square-free monomial ideals via facet ideals: a survey. Contemp. Math. 448 (2007), 91-117. 3

[34] H.T. Hà, A. Van Tuyl, Splittable ideals and the resolutions of monomial ideals. J. Algebra 309 (2007), no. 1, 405-425. $19,22,37,38$

[35] J. He, A. Van Tuyl, Algebraic properties of the path ideal of a tree. Comm. Algebra 38 (2010), 1725-1742. 13

[36] J. Herzog, T. Hibi, Monomial Ideals. GTM 260, Springer, 2011. 3, 12,16 
[37] J. Herzog, T. Hibi, F. Hreinsdóttir, T. Kahle, J. Rauh, Binomial edge ideals and conditional independence statements. Adv. in Appl. Math. 45 (2010), no. 3, 317-333. 13

[38] J. Herzog, T. Hibi, X. Zheng, Monomial ideals whose powers have a linear resolution. Math. Scand. 95 (2004), no. 1, 23-32. 22

[39] T. Hibi, K. Kimura, S. Murai, Betti numbers of chordal graphs and $f$-vectors of simplicial complexes. J. Algebra 323 (2010), no. 6, 1678-1689. 22

[40] A. Hoefel, G. Whieldon, Linear Quotients of the Square of the Edge Ideal of the Anticycle. Preprint (2011) arXiv:1106.2348v2 23

[41] N. Horwitz, Linear resolutions of quadratic monomial ideals. J. Algebra 318 (2007), no. 2, 981-1001. 23

[42] S. Hosten, G.G. Smith, Monomial Ideals. In Computations in algebraic geometry with Macaulay 2, Algorithms and Computations in Mathematics 8, pp. 73-100, Springer-Verlag, New York, 2001. 30

[43] M. Katzman, Characteristic-independence of Betti numbers of graph ideals. J. Combin. Theory Ser. A 113 (2006), no. 3, 435-454. 14

[44] D. Kiani, S. Moradi, Bounds for the regularity of edge ideal of vertex decomposable and shellable graphs. Preprint (2010) arXiv: 1007.4056v123

[45] K. Kimura, Non-vanishingness of Betti numbers of edge ideals. Preprint (2011) arXiv:1110.2333v3 22

[46] M. Kummini, Regularity, depth and arithmetic rank of bipartite edge ideals. J. Algebraic Combin. 30 (2009), no. 4, 429-445. 23

[47] S. Jacques, Betti Numbers of Graph Ideals. PhD Thesis, University of Sheffield, (2004). arXiv: math/0410107v1 39

[48] J. Martinez-Bernal, S. Morey, R. Villarreal, Associated primes of powers of edge ideals. Preprint (2011) arXiv: 1103.0992v324, 29

[49] E. Miller, B. Sturmfels, Combinatorial Commutative Algebra. GTM 227, Springer-Verlag, New York, 2004. 3, 14,27

[50] S. Morey, R. Villarreal, Edge ideals: algebraic and combinatorial properties. Preprint (2010). arXiv: $1012.5329 \mathrm{v} 3,3,23$

[51] S. Morey, E. Reyes, R. Villarreal, Cohen-Macaulay, shellable and unmixed clutters with a perfect matching of König type. J. Pure Appl. Algebra 212 (2008), no. 7, 1770-1786. 13

[52] E. Nevo, Regularity of edge ideals of $C_{4}$-free graphs via the topology of the lcm-lattice. J. Combin. Theory Ser. A 118 (2011), no. 2, 491-501, 16, 23

[53] E. Nevo, I. Peeva, Linear resolutions of powers of edge ideals. Preprint (2010). 22, 39

[54] I. Peeva, Graded Syzygies. Springer, 2010. 3, 14, 17, 23

[55] E. Scheinerman, D. Ullman, Fractional graph theory. A rational approach to the theory of graphs. John Wiley \& Sons, Inc., New York, 1997. 10

[56] R.Y. Sharp, Steps in Commutative Algebra (2nd Edition). Cambridge University Press, 2000. 3

[57] A. Simis, W. Vasconcelos, R.H. Villarreal, On the ideal theory of graphs. J. Algebra 167 (1994), no. 2, 389-416. 2

[58] R. Stanley, Combinatorics and Commutative Algebra, 2nd Ed. Birkhauser, Boston, 1996. 10, 11

[59] R. Stanley, The Upper Bound Conjecture and Cohen-Macaulay rings. Studies in Applied Math. 54 (1975), 135-142. 2

[60] B. Sturmfels, S. Sullivant, Combinatorial secant varieties. Pure Appl. Math. Q. 2 (2006), no. 3, part 1, 867-891. 28

[61] G. Valla, Betti numbers of some monomial ideals. Proc. Amer. Math. Soc. 133 (2005), no. 1, 57-63. 22

[62] A. Van Tuyl, Sequentially Cohen-Macaulay bipartite graphs: vertex decomposability and regularity. Arch. Math. (Basel) 93 (2009), no. 5, 451-459. 23,39 
[63] R.H. Villarreal, Cohen-Macaulay graphs. Manuscripta Math. 66 (1990), no. 3, 277-293. 2, 5

[64] R.H. Villarreal, Monomial algebras. Monographs and Textbooks in Pure and Applied Mathematics, 238. Marcel Dekker, Inc., New York, 2001. 3, 13, 38

[65] G. Whieldon, Jump sequences of edge ideals. Preprint (2010). arXiv:1012.0108v1 23

[66] R. Woodroofe, Matchings, coverings, and Castelnuovo-Mumford regularity. Preprint (2010) arXiv: 1009.275623

[67] R. Woodroofe, Chordal and sequentially Cohen-Macaulay clutters. Electron. J. Combin. 18 (2011), no. 1, Paper 208. 21

[68] X. Zheng, Resolutions of facet ideals. Comm. Algebra 32 (2004), no. 6, 2301-2324. 23

Department of Mathematical Sciences, Lakehead University, Thunder Bay, ON P7B 5E1, CANADA

E-mail address: avantuyl@lakeheadu.ca

URL: http://flash.lakeheadu.ca/ avantuyl/ 